\title{
The mitotic crosslinking protein PRC1 acts as a mechanical dashpot to resist microtubule sliding
}

\author{
Ignas Gaska, Mason Armstrong, April Alfieri, Scott Forth ${ }^{\star}$ \\ Department of Biological Sciences \\ Rensselaer Polytechnic Institute \\ Troy, NY 12180, USA
}

*Correspondence addressed to: forths@rpi.edu 


\section{Abstract}

3 Cell division in eukaryotes requires the regulated assembly of the spindle apparatus. The

4 proper organization of microtubules within the spindle is driven by motor proteins that

5 exert forces to push and slide filaments, while non-motor proteins can crosslink filaments

6 into higher order motifs such as overlapping bundles. It has not been clear how active

7 and passive forces are integrated to produce regulated mechanical outputs within

8 spindles. Here we employ a combined optical tweezers and TIRF microscopy instrument

9 to directly measure the resistive forces produced by the mitotic crosslinking protein PRC1.

10 We observe that PRC1 generates frictional forces that resist microtubule sliding. These

11 forces scale with microtubule sliding velocity and the number of PRC1 crosslinks, but do

12 not depend on overlap length or PRC1 density within overlaps. Our results suggest that

13 PRC1 ensembles act like a mechanical dashpot, producing significant resistance against

14 fast motions, but minimal resistance against slow motions, allowing for the integration of

15 diverse motor activities into a single mechanical outcome. 


\section{Introduction}

Cell division in eukaryotes requires the organization of the microtubule-based mitotic spindle to segregate chromosomes and regulate positioning of the cell division plane (Kapoor, 2017). Within the spindle, subsets of microtubules are organized into specialized arrays whose filaments can undergo relative sliding (Mclntosh and Hays, 2016). Microtubule sliding is driven in part by motor proteins that either crosslink filaments or cluster at microtubule ends to exert active sliding or pulling forces along the filament (Forth and Kapoor, 2017). Single molecule analyses of diverse motor proteins found within spindles indicate that stepping rates can vary over nearly several orders of magnitude, from dynein at nearly $\sim 1$ micron/sec (King and Schroer, 2000; McKenney et al., 2014; Ross et al., 2006) to various mitotic kinesins that range from 25-300 nm/sec (Braun et al., 2017; Drechsler et al., 2014; Kapitein et al., 2005; Reinemann et al., 2017, 2018; Wijeratne and Subramanian, 2018). Spindle microtubules can also be bundled into higher-order arrays by non-motor proteins that can sort filaments by polarity and regulate the relative motions of sliding microtubules (Subramanian and Kapoor, 2012). It has been proposed that crosslinking by such proteins may produce a 'brake-like' resistance to filament sliding that acts to balance motor-driven forces (Janson et al., 2007), leading to an integration of active and passive forces that sets parameters such as spindle length and filament sliding velocity.

Among the non-motor mitotic crosslinking proteins are members of the conserved MAP65 family, which include the yeast Ase1 and the human PRC1. These proteins have been shown to preferentially crosslink antiparallel microtubules both in vitro and within the anaphase central spindle microtubule array (Bieling et al., 2010; Janson et al., 2007; Rincon et al., 2017; Subramanian et al., 2010; Tikhonenko et al., 2016; Yamashita et al., 2005) and to help form "bridging fibers" to crosslink kinetochore microtubules during metaphase (Kajtez et al., 2016; Polak et al., 2017; Vukušić et al., 2017). Several lines of evidence suggest that microtubule arrays crosslinked by MAP65 proteins act as

45 mechanical linkages between segregating chromosomes and separating spindle halves 
47 density is reduced within spindle midzones in anaphase and the two spindle halves 48 appear fully detached from one another (Mollinari, 2004; Mollinari et al., 2002; Zhu et al., 49 2006) and chromosome segregation rates and separation distance both increase 50 (Pamula et al., 2019). Second, the yeast MAP65, Ase1, has been shown in in vitro assays 51 to slow motor-driven microtubule sliding via an adaptive braking mechanism (Braun et al., 52 2011) and can autonomously produce entropic expansion forces that prevent 53 microtubules from sliding apart (Lansky et al., 2015). In dividing yeast cells, Ase1 has 54 been shown to selectively stabilize longer bundles while still allowing for the motor-driven 55 transport of shorter microtubules, suggesting that Ase1 can produce an overlap length56 dependent resistance (Janson et al., 2007). Third, single molecule studies revealed that 57 monomeric PRC1 constructs containing just the microtubule-binding domain of the protein generate frictional resistance when moved along the microtubule lattice when under tension (Forth et al., 2014). However, it is currently unclear how ensembles of fulllength human PRC1 molecules crosslinking microtubules in micron-scale overlaps produce resistive forces. Specifically, it is unknown how resistive forces might be regulated within sliding bundles by parameters such as PRC1 density, microtubule overlap length, or relative sliding speed.

Here, using simultaneous TIRF microscopy and optical trapping methods, we directly measure the resistive forces generated by ensembles of PRC1 molecules crosslinking microtubule pairs. We find that the magnitude of resistive forces linearly scales both with the velocity at which microtubules slide apart and with the total number of engaged crosslinkers in overlap region, but not the length of the overlap or the density of crosslinks. Surprisingly, we observe that pausing during sliding allows the system to relieve tension and reinitiating sliding results in increased resistive forces, suggesting that upon cessation of relative sliding, PRC1 molecules can rearrange within the overlap to form a 73 higher-order configuration that more strongly resists microtubule separation. Finally,

74 using computational modeling, we describe how PRC1 can be condensed into shrinking 75 overlaps by microtubule ends that act as 'leaky' reflective barriers to diffusion. Together, 76 these results suggest that PRC1-crosslinked microtubule pairs generate viscous 77 resistance to act like a mechanical dashpot during filament sliding. 
Results

\section{Relative sliding of microtubules crosslinked by PRC1 generates resistive forces}

81 To characterize the mechanical properties of PRC1-mediated microtubule bundles, we 82 established an assay that allowed us to control microtubule sliding motions while 83 simultaneously measuring the forces between two crosslinked microtubules and observing the distribution of GFP-PRC1 molecules within microtubule overlap regions. To achieve this, we employed a strategy similar to one previously used to measure pushing forces by kinesin-5 ensembles (Shimamoto et al., 2015). Briefly, microtubules containing HiLyte-647 and biotinylated tubulin were immobilized on a passivated coverslip via Neutravidin linkages. GFP-PRC1 and rhodamine-labelled microtubules were introduced into the sample chamber, generating microtubule bundles with varying overlap lengths and amounts of PRC1 molecules in overlaps. Finally, polystyrene beads coated with truncated kinesin-1 construct were introduced, allowing for long-lived attachments to microtubules. These beads were then held in an optical trap and used to both apply and measure force along the length of the microtubule bundle (Figure 1A).

Moving the sample stage at a fixed velocity parallel to the microtubule bundle axis allowed for controlled separation of filaments. Images of each of the two microtubules and the GFP-PRC1 molecules were acquired using total internal reflection fluorescence (TIRF) microscopy. These data show the surface-immobilized microtubule moving at a constant velocity, the free microtubule held in place via the optically trapped bead, and the GFP. PRC1 molecules clustering into the region of decreasing overlap (Figure 1B). Upon complete separation of a microtubule pair, the free microtubule became detached from the bundle and freely swiveled, while a small number of remaining GFP-PRC1 molecules bound to the surface microtubule began to diffuse away from the microtubule end.

104 Simultaneously with the acquisition of the fluorescence imaging data, we recorded the force exerted on the bead using a custom-built force-calibrated optical tweezers instrument (Figure 1C). Before separating the microtubule pair, the measured force was approximately $0 \mathrm{pN}$. After a 10-second delay to confirm bead attachment and record the 
109 moved at a constant velocity and the magnitude of the force increased rapidly until 110 reaching a relatively stable value. Over the course of the pulling event, the force remained 111 nearly constant, with small and stochastic deviations around an average value. Upon 112 bundle separation, the force value sharply dropped to $0 \mathrm{pN}$, consistent with the 113 microtubules no longer being mechanically crosslinked. Together, these data 114 demonstrate that our instrument is capable of simultaneous force and fluorescence 115 measurement of sliding microtubule bundles moving at controllable velocities, and that 116 crosslinked microtubule pairs generate resistive forces when sliding.

We next examined the time-dependent distribution of GFP-PRC1 molecules within the overlap region throughout the experiment. Line scans of the GFP signal intensity were generated for each time point and plotted against distance along the microtubule positions

121 (Figure 1D and kymograph inset). We observed that at earlier time points, when the 122 overlap region is several microns in length, the GFP intensity is relatively evenly distributed within the overlap and has a mean value of 10 a.u. (blue line). As the overlap

124 decreased in length, the mean value of the intensity increased while the length distribution 125 became narrower, indicating that PRC1 molecules track the shrinking overlap region. 126 Once the overlap had reached a value close to 0 microns, the GFP intensity signal 127 reached a maximum value of $\sim 40$ a.u. (red line). Control experiments with non-moving 128 microtubule pairs reveal that loss of GFP fluorescence signal due to photobleaching 129 occurs at a rate of only $\sim 4 \%$ per minute, suggesting that time-dependent changes in 130 signal are due to changes in PRC1 concentration, and not photobleaching (Figure 131 S1).These data suggest that the PRC1 molecules are moving closer together within the 132 overlap, increasing in density as the overlap length decreases.

134 We next sought to determine what percentage of PRC1 molecules persisted within the 135 overlap during its reduction in length. We calculated the integrated GFP intensity within 136 overlaps, normalized both the integrated intensity and overlap lengths relative to their 137 initial values, and compared these relationships at four different pulling velocities (Figure 138 1E). Here, we observe that as the overlap length decreases, the percentage of remaining PRC1 molecules decreases. As the overlap length approached zero, approximately one 
140 third to one half of the original molecules can still be detected within the overlap. Together,

141 these data suggest that a subset of the initial population of PRC1 molecules is lost from

142 the bundle, but the molecules that remain are driven to cluster within the shrinking overlap

143 region between sliding microtubules.

The magnitude of resistive forces increases with increasing relative microtubule velocity

We next sought to determine how resistive forces generated during bundle disruption would depend on sliding velocity. We therefore disrupted microtubule bundles at four different velocities that are similar to reported stepping rates for various mitotic kinesins: $25 \mathrm{~nm} / \mathrm{s}, 50 \mathrm{~nm} / \mathrm{s}, 100 \mathrm{~nm} / \mathrm{s}$, and $200 \mathrm{~nm} / \mathrm{s}$. Inspection of the fluorescence data revealed that at each velocity, bundle overlap decreased uniformly at the rate of microtubule sliding, and PRC1 molecules consistently tracked and concentrated within the shrinking overlap (Figure 2A). Forces measured during bundle disruption events appeared to be generally larger in magnitude at higher sliding velocities (Figure 2B). We confirmed that the bead-microtubule attachments persisted throughout all pulls by noting that the overlap length reduction was continuous and no sudden force reductions to zero load were observed, as we saw in control cases where bead-microtubule attachment was transiently lost (Figure S2). We also confirmed that the microtubule-surface attachments remained robust throughout our experiments by measuring the velocity of the surface-immobilized microtubule and finding it to consistently match that of the controlled stage velocity (Figure

We repeated these experiments multiple times for each condition and calculated the average force during sliding events at each velocity (Figure $2 \mathrm{C}$ ), revealing a positive correlation between faster sliding velocity and increasing force. We next considered the relationship between force and overlap length for the same data sets. These data reveal

167 that the force trend throughout the entire sliding event remains relatively constant, 168 suggesting minimal correlation between force and overlap length (Figure 2D). After 169 calculating the slopes of these data (Figure 2D, dashed gray lines) and averaging multiple traces, we confirmed that the change in force with overlap length is close to $0 \mathrm{pN} / \mathrm{micron}$, 
171 but with a slightly negative trend (Figure 2E). This indicates that there may be a small

172 increase in force as the bundle approaches separation, but that the force is relatively

173 constant within a given pulling event and is largely independent of overlap length.

174 Previous analyses of microtubule bundles crosslinked by Ase1 molecules suggested that

175 as the density of crosslinkers increased, entropic forces that resisted bundle separation

176 rapidly grew in magnitude (Lansky et al., 2015). We observe a similar increase in the

177 density of PRC1 molecules as overlap lengths approach zero (Figure 2F). However,

178 analysis of the change in force values relative to change in overlap lengths for data

179 subsets selected between 0 and 0.5 microns (Figure 2D, solid grey lines) do not reveal a

180 sharp increase as overlap lengths approach 0 microns (Figure 2G), suggesting that the

181 resistance to microtubule pair separation produced by Ase1 and PRC1 operate by

182 different mechanisms.

\section{Resistive forces scale with number of PRC1 molecules but not overlap length}

While we observed a general trend of force values that increase with faster pulling velocities, we noted that there were individual instances where the force recorded during pulls at our slowest rate $(25 \mathrm{~nm} / \mathrm{s})$ exhibited higher average values than those measured during our fastest pulls $(200 \mathrm{~nm} / \mathrm{s}$ ) (Figure $2 \mathrm{C}$ ). This led us to examine additional parameters that may contribute to the production of resistive forces. The formation of bundles occurs spontaneously within the sample chamber, and therefore bundles can have both variable initial overlap lengths and different concentrations of GFP-PRC1. To allow for more direct comparisons across different pulling rates, we selected subsets from our data to perform analyses within a small range of overlap values (0.5-1.5 microns). We next plotted individual force and GFP integrated intensity data points from within this range for each velocity and found that forces increased with increasing integrated GFP intensity (Figure 3A). We next calculated the slopes of each constant sliding velocity data set and observed that the average slope value increased nearly linearly with increasing velocity (Figure 3B). Plotting the same force values against overlap length did not show 199 a significant correlation (Figure 3C), and the slopes of the force-overlap relationships 200 within this subset of data did not show a clear dependence on overlap length (Figure 3D). 201 Together, these data reveal that the magnitude of resistive force scales both with GFP 
202 integrated intensity, which is proportional to the total number of PRC1 molecules in the 203 overlap, as well as sliding velocity, but does not depend on the overlap geometry of the 204 system.

Pausing of sliding results in force relaxation, while resumption of sliding produces increased resistive forces

We occasionally encountered situations where attempts to slide bundles apart failed, due to insufficient force production from our trapping laser or bead detachment from the microtubule due to high loads generated despite no observable filament sliding. These events became more frequent the longer the sample sat on the microscope, leading us to hypothesize that PRC1 molecules within overlaps were undergoing a time-dependent rearrangement that was more resistant to microtubule sliding. To test this idea, we repeated our sliding experiments but introduced a short (20 second) pause in the microtubule motion before resuming the sliding event. Measured forces revealed three distinct regimes: an initial sliding event similar to what we'd previously observed, a relaxation event during the pause, and a second sliding event whose force magnitude and rate of force increase were larger than for the initial pull (Figure 4A). Fluorescence images reveal that the overlap region did not appreciably change in length, nor did the integrated GFP signal decrease during the pause (Figure 4B). These distinct behaviors

221 between the first and second pull were consistent across all bundles tested (Figure 4C).

222 During the pause, we observed a reduction in force that decayed to nearly zero pN after

$223 \sim 20$ seconds (Figure 4D). Normalizing the force values relative to the initial value at the 224 pause onset revealed a characteristic exponential decay whose time constant was similar 225 (7.5 +/- 0.2 seconds) for all bundles examined, regardless of the magnitude of the initial 226 force (Figure 4E). Finally, we quantified the mean force measured with each of the 227 constant sliding regimes (Figure 4F) and the rate of force increase as measured by the 228 slope of the force signal prior to reaching a plateau value (Figure 4G). For each of these 229 parameters, the values measured during the second pull were consistently higher relative 230 to the first pull. Calculating the ratio of these values revealed that the mean force and 231 force increase rate were nearly twice as large during the second pull relative to the first 232 (Figure 4H). Together these data suggest that allowing tension within the system to relax 
233 after sliding leads to a rearrangement of PRC1 molecules that is capable of producing

234 increased resistive forces upon re-initiation of constant-velocity sliding.

Computational modeling suggests that a partially reflective end barrier against PRC1 diffusion allows for sustained resistive forces

In order to gain insight into the mechanism by which PRC1 molecules produce resistive forces that scale with velocity but not overlap length, we turned to computational modeling. We expanded upon a previously reported simulation method (Shimamoto et al., 2015) that models two microtubules bundled by individual crosslinkers whose loaddependent behavior on the microtubule lattice can be defined by four parameters. First, an individual crosslinking molecule has two domains which are linked by a linear 'spring' whose stiffness is described by $\mathrm{k}_{\text {stiffness }}$ and which, when separated by a distance $\Delta \mathrm{x}$, can produce a force $F=\mathrm{k}_{\text {stiffness }}{ }^{*} \Delta \mathrm{x}$. Second, each of these domains can bind to and diffuse along the microtubule surface with a force-dependent rate $k_{\text {diffuse }}(F)$. Third, each microtubule-binding domain can detach from the microtubule lattice it contacts with a force-dependent rate constant described by $k_{\text {detach }}(F)$. Fourth, we introduced a parameter kend which describes the rate at which the molecule can diffuse off of the microtubule end once it reaches the last available lattice site on the microtubule (Figure 5A). Finally, we introduced the constraint that a molecule cannot move into a site that is already occupied by another crosslinking molecule. The simulation could then be run with a variable number of initial crosslinking molecules, overlap length, and rate of relative microtubule

254 sliding.

We first sought to explore the model's parameter space to determine which properties of PRC1 best describe our data. We first modulated kend over several orders of magnitude 258 and express this variation as ratio between $k_{e n d}$ and $k_{\text {diffuse. }}$ For small values of $k_{e n d}$ (e.g. $\left.259 \mathrm{k}_{\text {end }}=\mathrm{k}_{\text {diffuse }} / 500\right)$, molecules of PRC1 remained highly clustered and localized near 260 microtubule tips, which acted as reflective barriers against diffusion, producing extremely 261 large forces as overlap length decreased to zero. In contrast, larger values of kend (e.g. $262 \mathrm{k}_{\mathrm{end}}=\mathrm{k}_{\text {diffuse }} / 10$ ) resulted in molecules rapidly diffusing off microtubule ends into solution, 263 producing resistive forces that decreased to zero with decreasing overlap. (Figure 5B). 
264 An intermediate value of $\sim 50-100$ allowed us to reproduce the relatively flat force versus 265 overlap relationship (Figure 5C). We next varied both the diffusion and detachment rates, $266 \mathrm{k}_{\text {diffuse }}(\mathrm{F})$ and $\mathrm{k}_{\text {detach }}(\mathrm{F})$ respectively. For fast diffusion and detachment, we observed that 267 the forces generated were low and decreased towards zero pN (Figure 5D). As the 268 diffusion rate and detachment rate values were increased, we found that resistive forces 269 increased and the slope of the force relative to overlap length became shallower (Figure $2705 \mathrm{E}$ ). From these simulations, we conclude that the magnitude of resistive force and 271 dependence on parameters such as number of crosslinking molecules and overlap length

272 is highly sensitive to the rate at which molecules will diffuse both within the overlap and 273 off of the microtubule ends, as well as how likely they are to detach from the lattice under 274 load.

Once an optimal range of parameters had been identified, we next sought to determine 277 whether this simple set of conditions was sufficient to recapitulate the velocity- and crosslinker number-dependent resistive force generation behavior that we had observed experimentally. We performed simulations over a range of relative microtubule sliding velocities and determined the magnitude of force as a function of engaged crosslinkers. We found a strong linear relationship between these parameters at all velocities measured (Figure 5F). Slopes from linear regressions on these relationships revealed that the magnitude of resistive force per PRC1 molecule increased linearly with the applied sliding rate (Figure 5G). Together, these results reveal that a simple set of rates that include diffusion on the microtubule lattice, detachment, and diffusion off of microtubule ends are sufficient to describe how resistive forces within sliding bundles crosslinked by PRC1 are generated and depend on sliding velocity and total PRC1 concentration, and correlate well with our experimental measurements. 


\section{Discussion}

296 Our data reveal that ensembles of PRC1 molecules can produce resistive forces against 297 relative microtubule sliding. The magnitude of these forces scales with both the rate of 298 sliding and the total number of engaged crosslinks, but not crosslinker density or overlap 299 length (Figure 5H). The linearly proportional dependence of resistive forces on the sliding velocity suggests that PRC1 ensembles behave as viscous crosslinkers. Upon cessation of sliding the resistive force relaxes, while resumption of sliding results in resistive forces whose magnitude is higher than during the initial sliding event. Finally, we present a simple quantitative model that recapitulates key features of our data by introducing a parameter that allows for microtubule ends to act as partially reflective barriers against PRC1 diffusion.

Based on our results, we suggest that PRC1 ensembles act as a dashpot against microtubule separation. Dashpots are mechanical devices that produce viscous resistance to motions and whose force magnitude is proportional to velocity. We propose that this property may allow sliding microtubules within the spindle midzone to differentially resist motor proteins whose natural stepping rates can span a wide range.

312 For example, recent evidence suggests that microtubule minus-ends can recruit dynein motor proteins via the non-motor MAP NuMA (Charlebois et al., 2011; Elting et al., 2014, 2017). Dynein has been shown to step at rates $\sim 1$ micron/second in vitro; if it were to step

315 at this rate while pulling on microtubule minus-ends, the resistive forces experienced by the motors from the PRC1-mediated overlap would be large. In contrast, motor proteins

317 that localize within overlapping bundles within the spindle midzone, such as kinesin-5 or 318 kinesin-4, step at rates of $25-300 \mathrm{~nm} / \mathrm{s}$. At these velocities, PRC1 ensembles would 319 produce a lower resistance. Indeed, experiments performed with mixtures of PRC1 and 320 kinesin-5 suggest that PRC1 crosslinks do not substantially slow relative filament sliding 321 (Subramanian et al., 2010).

323 Recent reports suggest that the yeast MAP65, Ase1, is capable of generating significant 324 forces via an entropic mechanism as molecules are condensed into small overlaps 325 (Lansky et al., 2015). We find that PRC1 does not exhibit this behavior. What might 
326 account for the difference between these two similar types of crosslinking molecule? First,

327 the lifetime of Ase1 within overlaps is nearly at least 10-fold longer than PRC1 molecules

328 (Pamula et al., 2019; Schuyler et al., 2003). Second, our computational models

329 recapitulate observed sliding mechanics when the end-diffusion parameter is $50-100$

330 times smaller in magnitude than the lattice diffusion parameter. Decreasing the end-

331 diffusion constant shifts simulated crosslinker behavior to a pattern more similar to Ase1,

332 where crosslinkers can elastically return a partially slid bundle back to its original position

333 via entropic pressure. This suggests that the end-diffusion parameter is the basis of the

334 mechanistic difference between PRC1 and Ase1 bundles. While the authors analogize

335 Ase1's behavior within overlaps to that of a one-dimensional gas compressed via a

336 piston-like mechanism (Lansky et al., 2015), we propose that PRC1 behaves as if it were

337 compressed by a 'leaky' piston, where molecules can be scooped by the reflective barrier

338 of microtubule plus-ends within the shrinking overlap to generate higher packing

339 densities, but a significant fraction of PRC1 molecules do diffuse off the microtubule ends,

340 thereby reducing the contribution from entropic pressure.

342 We observe that pausing relative microtubule sliding results in a reduction of resistive 343 forces. Upon resumption of sliding, the rate at which force builds up as well as the 344 magnitude of resistive force during the sliding event both increase. We suggest that the 345 dissipation of force during the pause is due to the PRC1 molecules undergoing diffusion 346 within the overlap to relieve the tension built up across individual molecules. These 347 molecules diffuse towards microtubule crosslinking configurations wherein the relative 348 distance between binding sites is minimized, thus minimizing the force contribution. Why 349 might resumption of sliding after this molecular rearrangement result in higher forces? 350 We propose that within the overlaps and given sufficient time, a subset of PRC1 351 molecules might diffuse into adjacent sites and form higher order complexes. Evidence 352 for multimerization of crosslinkers has been shown for Ase1 diffusing within microtubule 353 overlaps (Kapitein et al., 2008). In these experiments, diffusive Ase1 molecules will 354 undergo a random walk within overlap until colliding with nearby Ase1 molecules, at which 355 point a brighter cluster with a slower diffusion rate is observed, suggesting two or more 356 molecules have interacted to form a type of complex. We propose that a similar 
357 mechanism might be at play in our assays, where multiple PRC1 molecules within non-

358 moving bundles could interact to form higher order assemblies which would likely 359 contribute to the observed higher resistive forces. Recent high-resolution analyses of 360 midzone formation reveal that shrinking microtubule overlaps appear to slow down 361 throughout anaphase, nearly stopping their motion even while spindle poles still separate 362 (Pamula et al., 2019). We hypothesize that were higher-order PRC1 clusters to form 363 during this slow-down or pausing phase, it would lead to an increased resistive load 364 against further microtubule sliding, thereby contributing to the stabilization and maintenance of a robust spindle midzone bundle.

Together our results elucidate mechanisms by which ensembles of PRC1 molecules can regulate the speed of microtubule motions within dividing cells. It will be interesting to see if other non-motor proteins that have been shown to bundle and cluster spindle or kinetochore microtubules, such as NuMA or HURP, produce similar viscous forces against microtubule sliding, or whether an alternate mechanism is employed. It will

372 similarly be interesting to determine whether similar mechanical dashpots are utilized 373 within other cellular processes, such as during remodeling of the actin cytoskeleton.

\section{Acknowledgements}

377 We thank Dr. Susan Gilbert for the kind gift of the kinesin-1 K439 plasmid and Dr. Blanca 378 Barquera for assistance with construct design. We wish to thank Dr. Brandon Bensel for 379 assistance with the optical trap operation. We thank members of the Gilbert and Bentley 380 labs at RPI, as well as Dr. Radhika Subramanian (MGH) and Dr. Tarun Kapoor 381 (Rockefeller) for helpful discussions, and members of the Forth lab for critical reading of 382 the manuscript. The authors declare no competing financial interests. Author 383 contributions: I.G., A.A. and S.F designed experiments; I.G. and A.A. collected data; M.A. 384 and S.F. designed and performed simulations; I.G. and S.F. wrote manuscript. Funding 385 was provided by RPI School of Science start-up funds to S.F. 


\section{References}

Bieling, P., Telley, I.A., and Surrey, T. (2010). A minimal midzone protein module controls formation and length of antiparallel microtubule overlaps. Cell 142, 420-432.

Braun, M., Lansky, Z., Fink, G., Ruhnow, F., Diez, S., and Janson, M.E. (2011). Adaptive braking by Ase1 prevents overlapping microtubules from sliding completely apart. Nat. Cell Biol. 13, 1259-1264.

Braun, M., Lansky, Z., Szuba, A., Schwarz, F.W., Mitra, A., Gao, M., Lüdecke, A., Ten Wolde, P.R., and Diez, S. (2017). Changes in microtubule overlap length regulate kinesin-14-driven microtubule sliding. Nat. Chem. Biol. 13, 1245-1252.

Charlebois, B.D., Kollu, S., Schek, H.T., Compton, D.A., and Hunt, A.J. (2011). Spindle pole mechanics studied in mitotic asters: Dynamic distribution of spindle forces through compliant linkages. Biophys. J. 100, 1756-1764.

Drechsler, H., McHugh, T., Singleton, M.R., Carter, N.J., and McAinsh, A.D. (2014). The Kinesin-12 Kif15 is a processive track-switching tetramer. Elife 2014.

Elting, M.W., Hueschen, C.L., Udy, D.B., and Dumont, S. (2014). Force on spindle microtubule minus ends moves chromosomes. J. Cell Biol. 206, 245-256.

Elting, M.W., Prakash, M., Udy, D.B., and Dumont, S. (2017). Mapping Load-Bearing in the Mammalian Spindle Reveals Local Kinetochore Fiber Anchorage that Provides Mechanical Isolation and Redundancy. Curr. Biol. 27, 2112-2122.e5.

Forth, S., and Kapoor, T.M. (2017). The mechanics of microtubule networks in cell division. J. Cell Biol. 216, 1525-1531.

Forth, S., Hsia, K.C., Shimamoto, Y., and Kapoor, T.M. (2014). Asymmetric friction of nonmotor MAPs can lead to their directional motion in active microtubule networks. Cell 157, 420-432.

Janson, M.E., Loughlin, R., Loïodice, I., Fu, C., Brunner, D., Nédélec, F.J., and Tran, P.T. (2007). Crosslinkers and Motors Organize Dynamic Microtubules to Form Stable Bipolar Arrays in Fission Yeast. Cell 128, 357-368.

413 Kajtez, J., Solomatina, A., Novak, M., Polak, B., Vukušić, K., Rüdiger, J., Cojoc, G., 414 Milas, A., Šumanovac Šestak, I., Risteski, P., et al. (2016). Overlap microtubules link sister k-fibres and balance the forces on bi-oriented kinetochores. Nat. Commun. 7, 11.

Kapitein, L.C., Peterman, E.J.G., Kwok, B.H., Kim, J.H., Kapoor, T.M., and Schmidt, C.F. (2005). The bipolar mitotic kinesin Eg5 moves on both microtubules that it crosslinks. Nature 435, 114-118.

Kapitein, L.C., Janson, M.E., van den Wildenberg, S.M.J.L., Hoogenraad, C.C.,

422 Kapoor, T. (2017). Metaphase Spindle Assembly. Biology (Basel). 6, 8.

423 King, S.J., and Schroer, T.A. (2000). Dynactin increases the processivity of the 424 cytoplasmic dynein motor. Nat. Cell Biol. 2, 20-24. 
Lansky, Z., Braun, M., Lüdecke, A., Schlierf, M., Ten Wolde, P.R., Janson, M.E., Diez, S., Ludecke, A., Schlierf, M., Ten Wolde, P.R., et al. (2015). Diffusible Crosslinkers Generate Directed Forces in Microtubule Networks. Cell 160, 1159-1168.

Mclntosh, J., and Hays, T. (2016). A Brief History of Research on Mitotic Mechanisms. Biology (Basel). 5, 55.

McKenney, R.J., Huynh, W., Tanenbaum, M.E., Bhabha, G., and Vale, R.D. (2014). Activation of cytoplasmic dynein motility by dynactin-cargo adapter complexes. Science (80-. ). 345, 337-341.

Mollinari, C. (2004). Ablation of PRC1 by Small Interfering RNA Demonstrates that Cytokinetic Abscission Requires a Central Spindle Bundle in Mammalian Cells, whereas Completion of Furrowing Does Not. Mol. Biol. Cell 16, 1043-1055.

Mollinari, C., Kleman, J.P., Jiang, W., Schoehn, G., Hunter, T., and Margolis, R.L. (2002). PRC1 is a microtubule binding and bundling protein essential to maintain the mitotic spindle midzone. J. Cell Biol. 157, 1175-1186.

Pamula, M.C., Carlini, L., Forth, S., Verma, P., Suresh, S., Legant, W.R., Khodjakov, A., Betzig, E., and Kapoor, T.M. (2019). High-resolution imaging reveals how the spindle midzone impacts chromosome movement. J. Cell Biol. 218, 2529-2544.

Polak, B., Risteski, P., Lesjak, S., and Tolić, I.M. (2017). PRC 1-labeled microtubule bundles and kinetochore pairs show one-to-one association in metaphase. EMBO Rep. 18, 217-230.

Reinemann, D.N., Sturgill, E.G., Das, D.K., Degen, M.S., Vörös, Z., Hwang, W., Ohi, R., and Lang, M.J. (2017). Collective Force Regulation in Anti-parallel Microtubule Gliding by Dimeric Kif15 Kinesin Motors. Curr. Biol. 27, 2810-2820.e6.

Reinemann, D.N., Norris, S.R., Ohi, R., and Lang, M.J. (2018). Processive Kinesin-14 HSET Exhibits Directional Flexibility Depending on Motor Traffic. Curr. Biol.

Rincon, S.A., Lamson, A., Blackwell, R., Syrovatkina, V., Fraisier, V., Paoletti, A., Betterton, M.D., and Tran, P.T. (2017). Kinesin-5-independent mitotic spindle assembly requires the antiparallel microtubule crosslinker Ase1 in fission yeast. Nat. Commun. 8.

Ross, J.L., Wallace, K., Shuman, H., Goldman, Y.E., and Holzbaur, E.L.F. (2006). Processive bidirectional motion of dynein-dynactin complexes in vitro. Nat. Cell Biol. 8, 562-570.

Schuyler, S.C., Liu, J.Y., and Pellman, D. (2003). The molecular function of Ase1p: Evidence for a MAP-dependent midzone-specific spindle matrix. J. Cell Biol. 160, 517528.

Shimamoto, Y., Forth, S., and Kapoor, T.M. (2015). Measuring Pushing and Braking Forces Generated by Ensembles of Kinesin-5 Crosslinking Two Microtubules. Dev. Cell $34,669-681$.

Subramanian, R., and Kapoor, T.M. (2012). Building Complexity: Insights into SelfOrganized Assembly of Microtubule-Based Architectures. Dev. Cell 23, 874-885.

Subramanian, R., Wilson-Kubalek, E.M., Arthur, C.P., Bick, M.J., Campbell, E.A., Darst, 
S.A., Milligan, R.A., and Kapoor, T.M. (2010). Insights into antiparallel microtubule crosslinking by PRC1, a conserved nonmotor microtubule binding protein. Cell 142, 433-443.

Tikhonenko, I., Irizarry, K., Khodjakov, A., and Koonce, M.P. (2016). Organization of microtubule assemblies in Dictyostelium syncytia depends on the microtubule crosslinker, Ase1. Cell. Mol. Life Sci. 73, 859-868.

Vukušić, K., Buđa, R., Bosilj, A., Milas, A., Pavin, N., and Tolić, I.M. (2017). Microtubule Sliding within the Bridging Fiber Pushes Kinetochore Fibers Apart to Segregate Chromosomes. Dev. Cell 43, 11-23.e6.

Wijeratne, S., and Subramanian, R. (2018). Geometry of antiparallel microtubule bundles regulates relative sliding and stalling by PRC1 and kif4A. Elife 7.

Woll, K.A., Guzik-Lendrum, S., Bensel, B.M., Bhanu, N. V., Dailey, W.P., Garcia, B.A., Gilbert, S.P., and Eckenhoff, R.G. (2018). An allosteric propofol-binding site in kinesin disrupts kinesin-mediated processive movement on microtubules. J. Biol. Chem. 293, 11283-11295.

Yamashita, A., Sato, M., Fujita, A., Yamamoto, M., and Toda, T. (2005). The Roles of Fission Yeast Ase1 in Mitotic Cell Division, Meiotic Nuclear Oscillation, and Cytokinesis Checkpoint Signaling. Mol. Biol. Cell 16, 1378-1395.

Zhu, C., Lau, E., Schwarzenbacher, R., Bossy-Wetzel, E., and Jiang, W. (2006). Spatiotemporal control of spindle midzone formation by PRC1 in human cells. Proc. Natl. Acad. Sci. U. S. A. 103, 6196-6201. 


\section{Figure Legends}

488 Figure 1. Direct measurement of force across sliding microtubules crosslinked by 489 PRC1

490 (A) Schematic of the assay. A surface-immobilized microtubule (labelled with HiLyte-647 491 tubulin and biotinylated-tubulin, purple) is crosslinked via GFP-PRC1 molecules to a 492 second microtubule (labelled with rhodamine-tubulin, red) which is held via a kinesin493 coated bead in an optical trap. (B) Time series of fluorescent images acquired via TIRF 494 microscopy during microtubule sliding event. GFP-PRC1 and individual microtubule 495 channels are shown along with composite image. Scale bar $=4$ microns. (C) 496 Representative force time series data (raw, gray; averaged, red) during microtubule 497 sliding. Initiation of pull at 10 s and disruption event at $\sim 63$ s are labeled. (D) Linescan 498 analysis from GFP channel. GFP intensities at select time points are plotted against 499 length, with corresponding time points noted on kymograph inset (blue $=5 \mathrm{~s}$, green $=15 \mathrm{~s}$, 500 yellow $=25 \mathrm{~s}$, orange $=35 \mathrm{~s}$, red $=45 \mathrm{~s})$. Kymograph scale bars: vertical $=10 \mathrm{~s}$, horizontal

$501=2$ microns. (E) GFP signals within overlap regions were integrated and normalized to 502 their values at the initial overlap and are plotted against normalized overlap length. Data 503 from sliding events at four different velocities are shown ( $\mathrm{N}>8$ for each velocity, error bars $504=$ SD). 
bioRxiv preprint doi: https://doi.org/10.1101/2019.12.12.874529; this version posted December 13, 2019. The copyright holder for this preprint (which was not certified by peer review) is the author/funder, who has granted bioRxiv a license to display the preprint in perpetuity. It is made available under aCC-BY-NC-ND 4.0 International license.

\section{Figure 1}
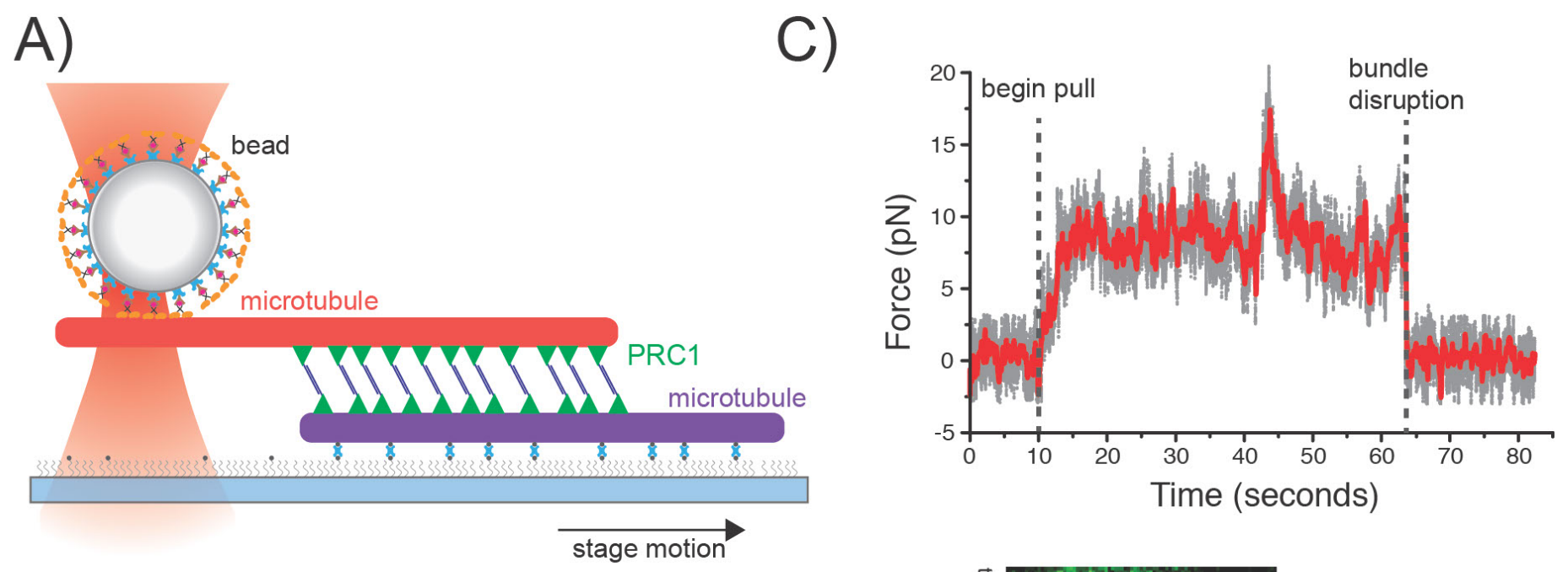

B)

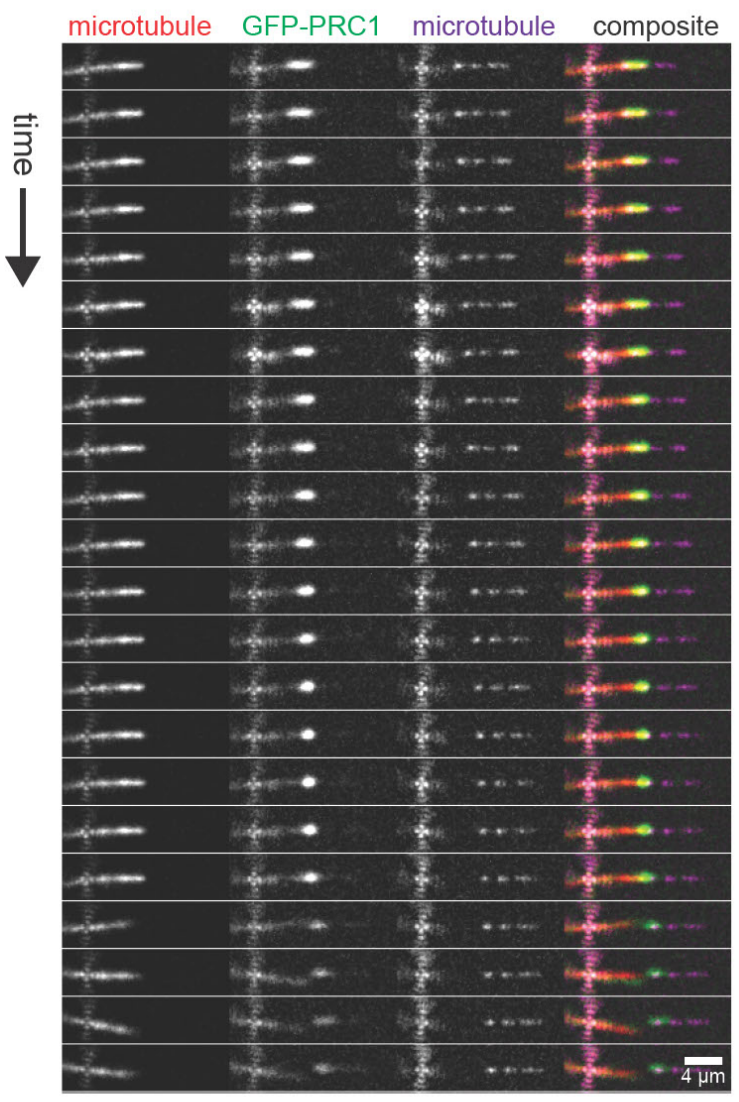

D)

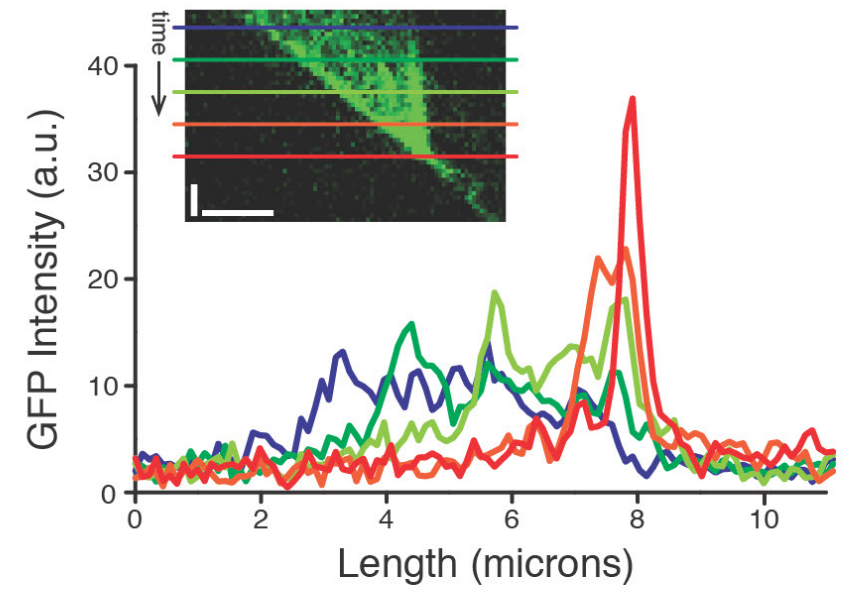

E)

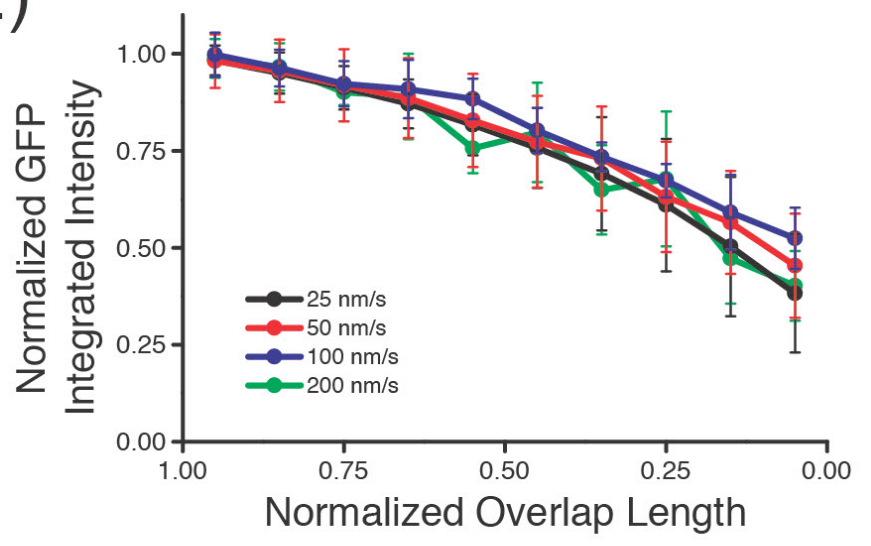


505 Figure 2. Resistive forces generated by PRC1 crosslinks scale with relative 506 microtubule sliding velocity

507 (A) Time series of composite fluorescent images acquired via TIRF microscopy during 508 microtubule sliding events at three different velocities: $50 \mathrm{~nm} / \mathrm{s}, 100 \mathrm{~nm} / \mathrm{s}$, and $200 \mathrm{~nm} / \mathrm{s}$. 509 Purple $=$ surface-immobilized microtubule, red $=$ bead-bound microtubule, green $=$ GFP510 PRC1. (B) Representative averaged force time series at four velocities (black $=25$, red $511=50$, blue $=100$, green $=200 \mathrm{~nm} / \mathrm{s}$ ). Disruption events are marked with vertical arrows.

512 (C) Box plots of mean force values during sliding events plotted for each velocity ( $\mathrm{N}>7$ for 513 all velocities). (D) Representative force trajectories plotted against measured bundle 514 overlap length at four different velocities, (black $=25$, red $=50$, blue $=100$, green $=200$ $515 \mathrm{~nm} / \mathrm{s}$ ). Shaded yellow region highlights bundle overlap lengths of 0-0.5 microns. Dashed 516 gray lines = linear fits for duration of entire bundle pull event. Solid gray lines = linear fits 517 for bundle overlap lengths of 0-0.5 microns. (E) Box plots of slopes calculated from force 518 versus total overlap length ( $N>9$ for all velocities). (F) GFP Density values normalized to 519 their values at the initial overlap are plotted against normalized overlap length. Data from 520 all events were binned in $200 \mathrm{~nm}$ length increments and averaged for each velocity. ( $N>7$ 521 for all velocities, error bars $=S E$ ). (G) Box plots of slopes calculated from force versus 522 overlap lengths for data selected between $0-0.5$ microns of overlap length ( $\mathrm{N}>7$ for all 523 velocities). 
bioRxiv preprint doi: https://doi.org/10.1101/2019.12.12.874529; this version posted December 13, 2019. The copyright holder for this preprint (which was not certified by peer review) is the author/funder, who has granted bioRxiv a license to display the preprint in perpetuity. It is made available under aCC-BY-NC-ND 4.0 International license.

\section{Figure 2}
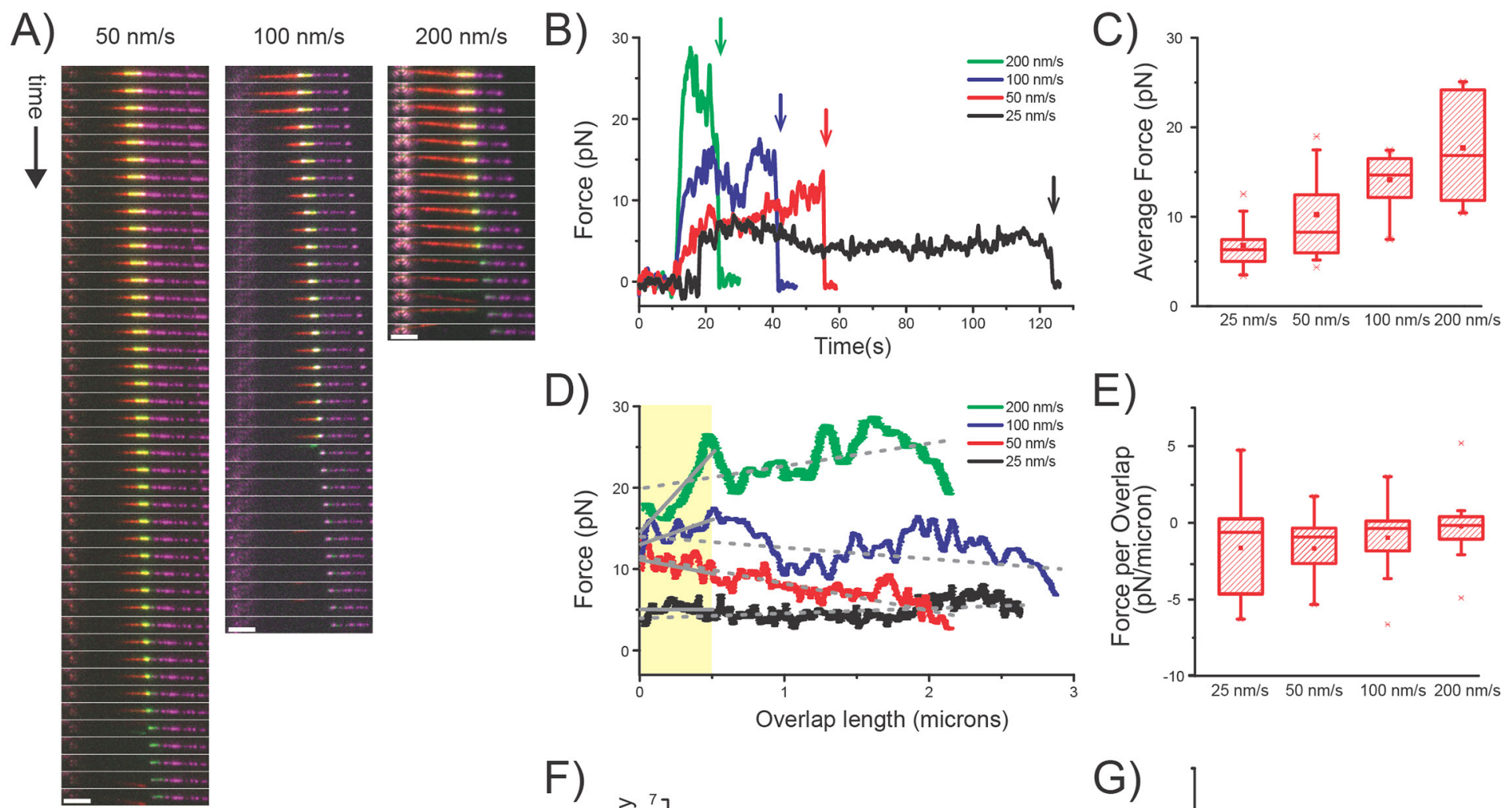

D)

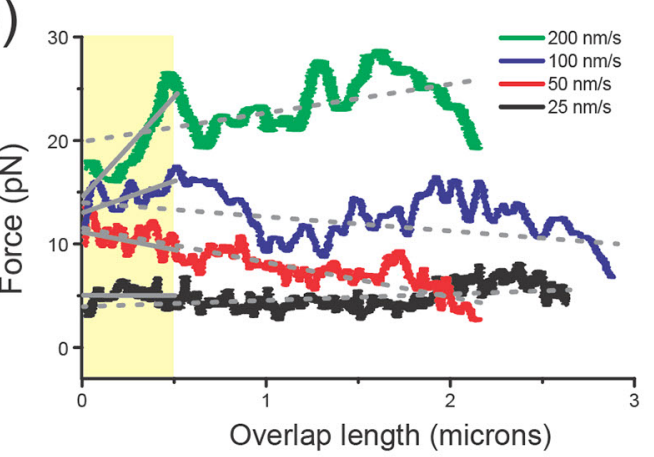

E)

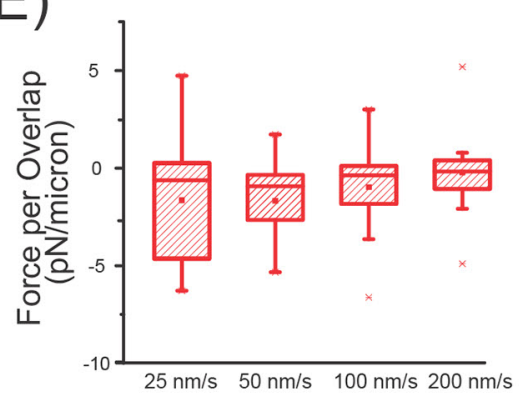

F)
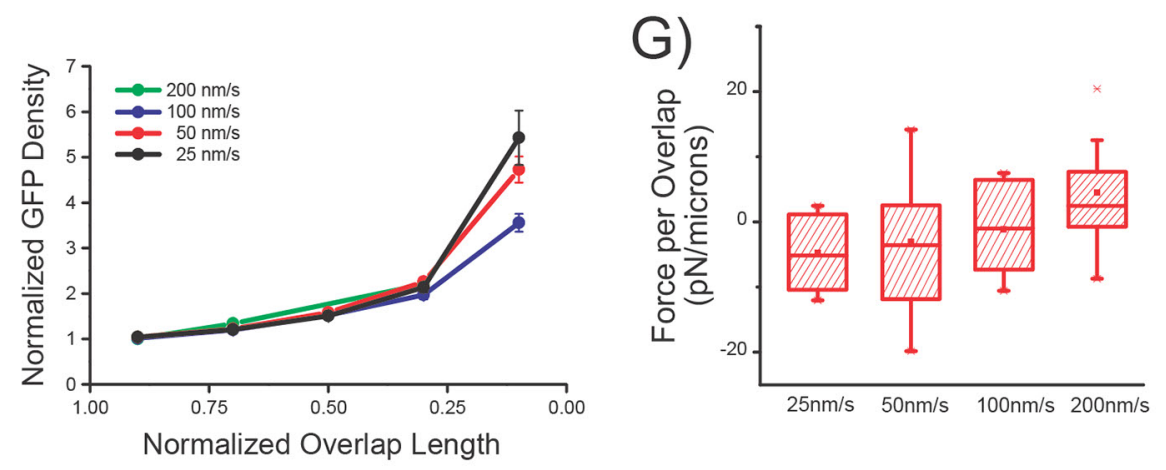


\section{Figure 3. Resistive forces are proportional to PRC1 crosslink number but not}

525 overlap length

526 (A) Integrated GFP intensity values within overlaps and the corresponding average force 527 during image acquisition period. Data from overlaps between 0.5 and 1.5 microns from 528 all traces are plotted (Points: black $=25 \mathrm{~nm} / \mathrm{s}$, red $=50 \mathrm{~nm} / \mathrm{s}$, blue $=100 \mathrm{~nm} / \mathrm{s}$, green $=$ $529200 \mathrm{~nm} / \mathrm{s}$. Solid lines = linear fits to each data set). (B) Slopes of linear fits from (A). (C) 530 Same averaged force data points from (A), plotted against corresponding overlap length 531 (Points: black $=25 \mathrm{~nm} / \mathrm{s}$, red $=50 \mathrm{~nm} / \mathrm{s}$, blue $=100 \mathrm{~nm} / \mathrm{s}$, green $=200 \mathrm{~nm} / \mathrm{s}$. Solid lines $532=$ linear fits to each data set). (D) Slopes of linear fits from (C). $N>8$ for all velocities. 
bioRxiv preprint doi: https://doi.org/10.1101/2019.12.12.874529; this version posted December 13, 2019. The copyright holder for this preprint (which was not certified by peer review) is the author/funder, who has granted bioRxiv a license to display the preprint in perpetuity. It is made available under aCC-BY-NC-ND 4.0 International license.

Figure 3

A)

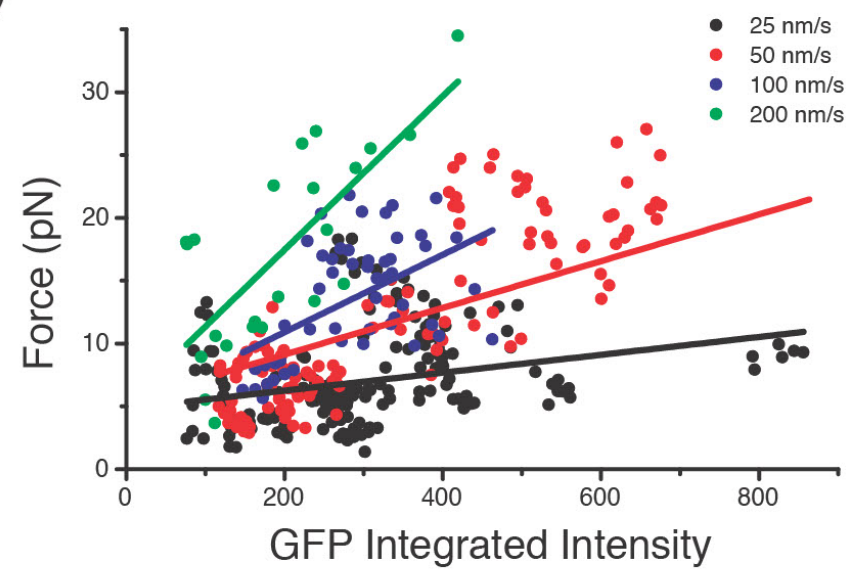

C)

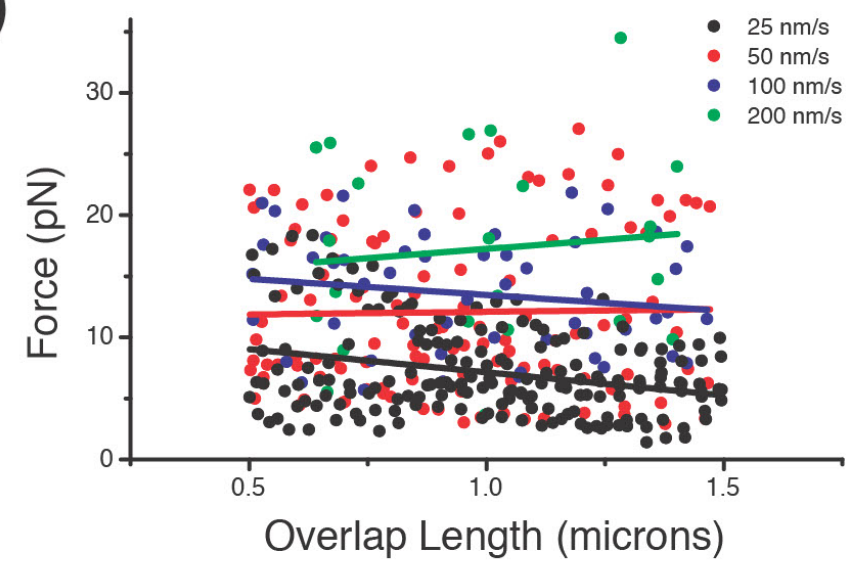

B)

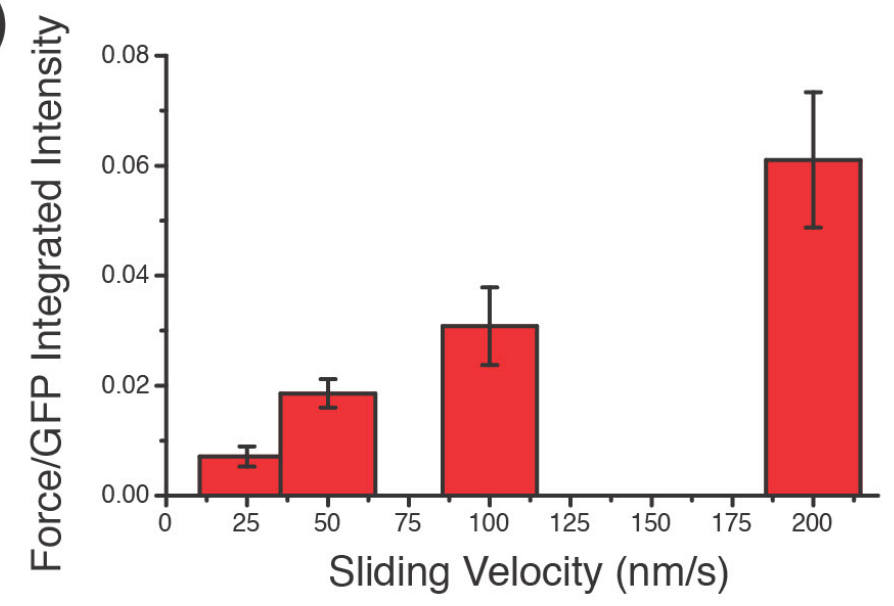

D)

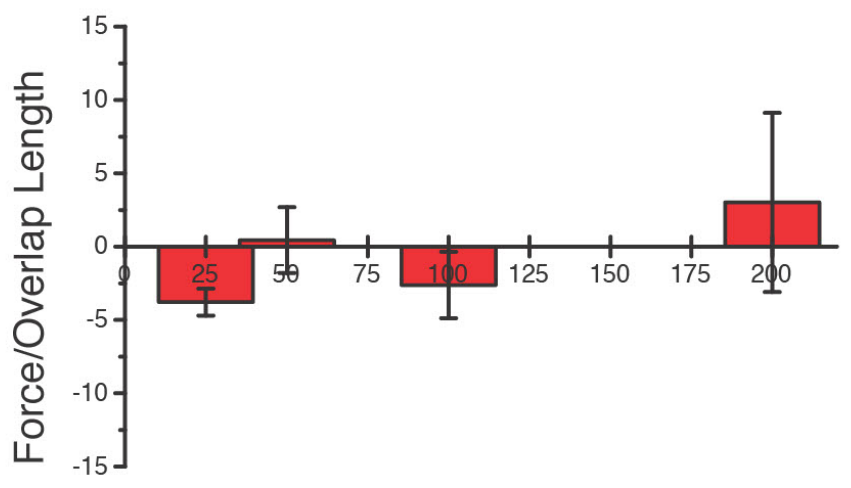

Sliding Velocity $(\mathrm{nm} / \mathrm{s})$ 
533 Figure 4. Pausing and then reinitiating sliding results in increased force production 534 after relaxation

535 (A) Representative force time series for double-pull assay. Shown are force and 536 microtubule positions during initial pull (red shading), 20 second pause (green shading),

537 and second pull (blue shading). (B) Kymograph of GFP fluorescence signal during double

538 pull experiment (vertical scale bar $=20$ seconds; horizontal scale bar $=1$ micron). (C)

539 Example initial and second pull force time series for three different bundles. Red $=$ initial 540 pull, Blue $=$ second pull. (D) Force time series during pause. Absolute force data are

541 shown ( $N=9$ independent bundles). (E) Normalized force time series during pause. Red

542 line $=$ exponential decay best fit to all data. Characteristic time $=7.5+/-0.2$ seconds. $(F)$

543 Mean force calculated during initial and second sliding events. $(G)$ Rate of force increase

544 calculated from linear fit of force versus time during first 5 seconds of pull for initial and

545 second pulling event. $(H)$ Ratio of values calculated in $(F)$ and $(G)$ for initial and second 546 pulling event ( $\mathrm{N}=9$ pull/pause/pull events). 


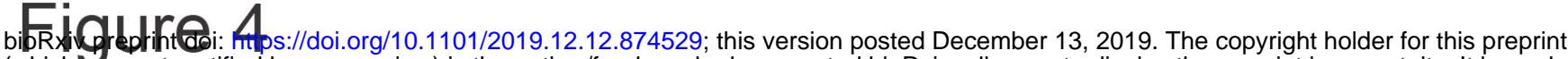
(which was not certified by peer review) is the author/funder, who has granted bioRxiv a license to display the preprint in perpetuity. It is made available under aCC-BY-NC-ND 4.0 International license.

A)
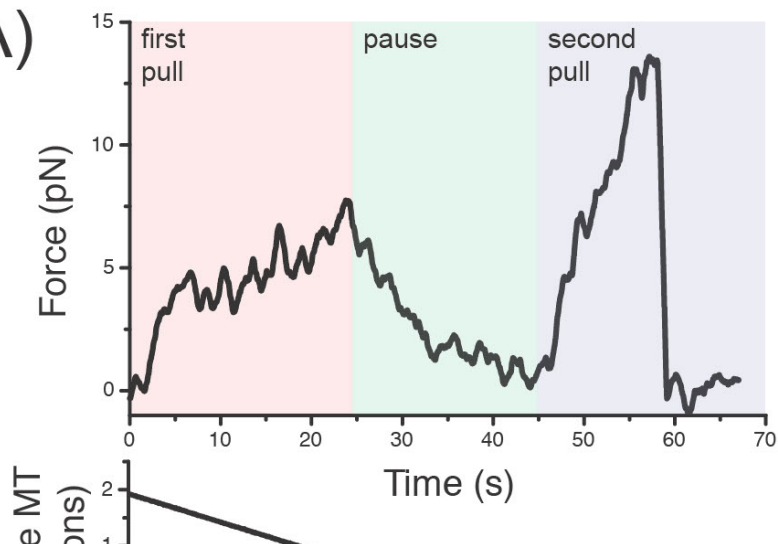

U

$\stackrel{\overparen{t}}{\frac{\pi}{J}}$

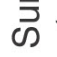

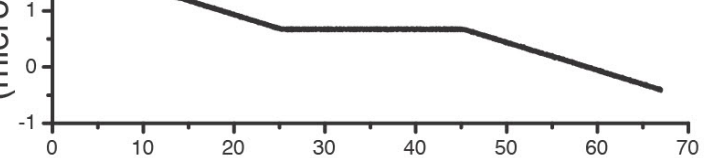

B)

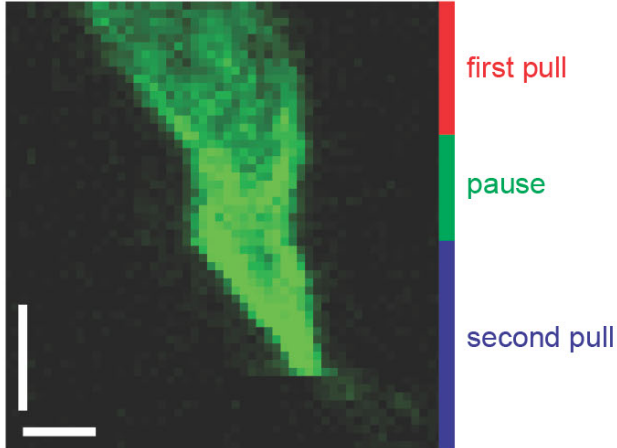

C)
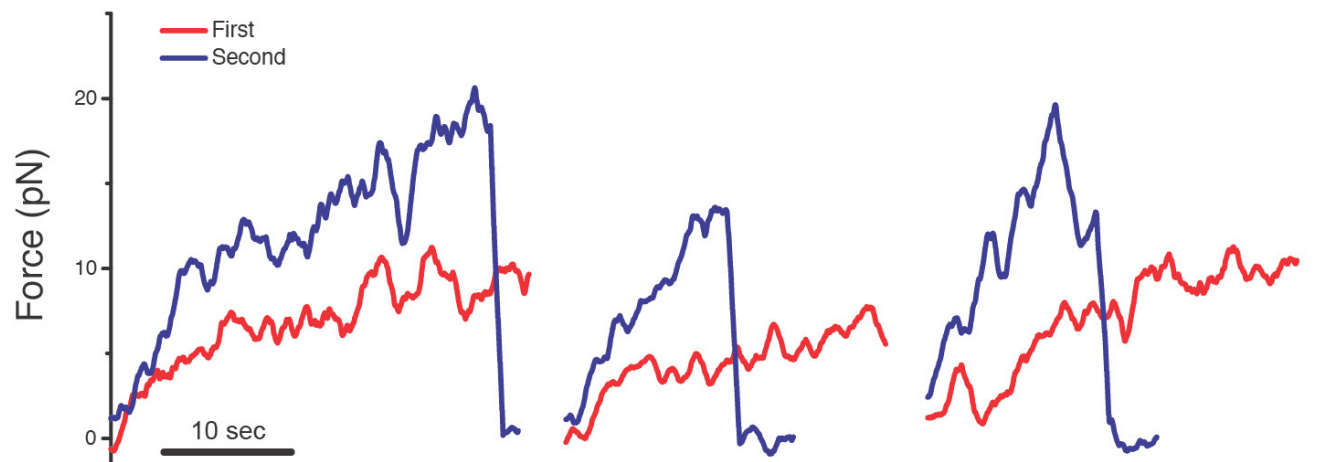

Time (s)

D)

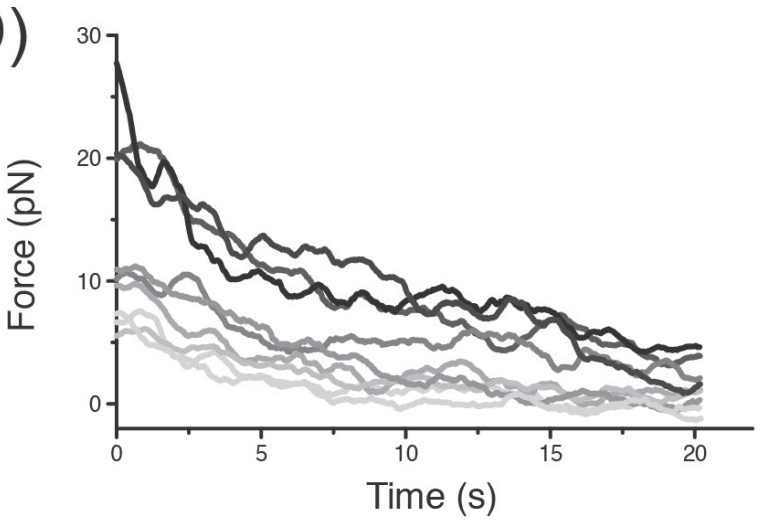

F)

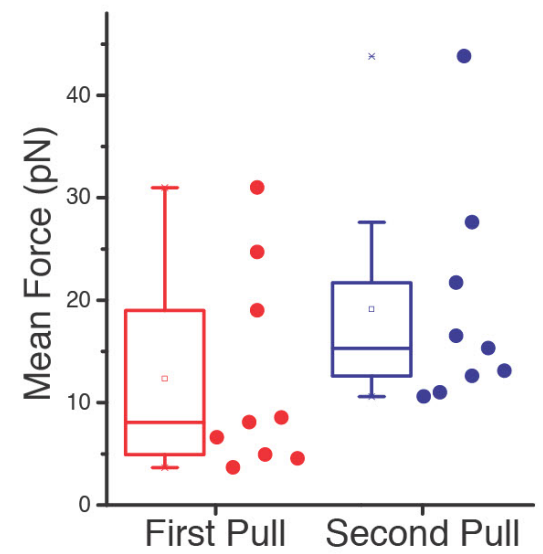

E)

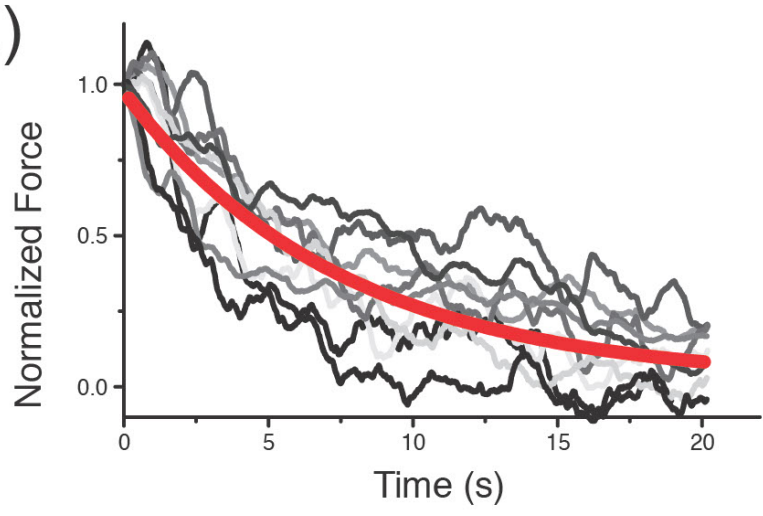

H)
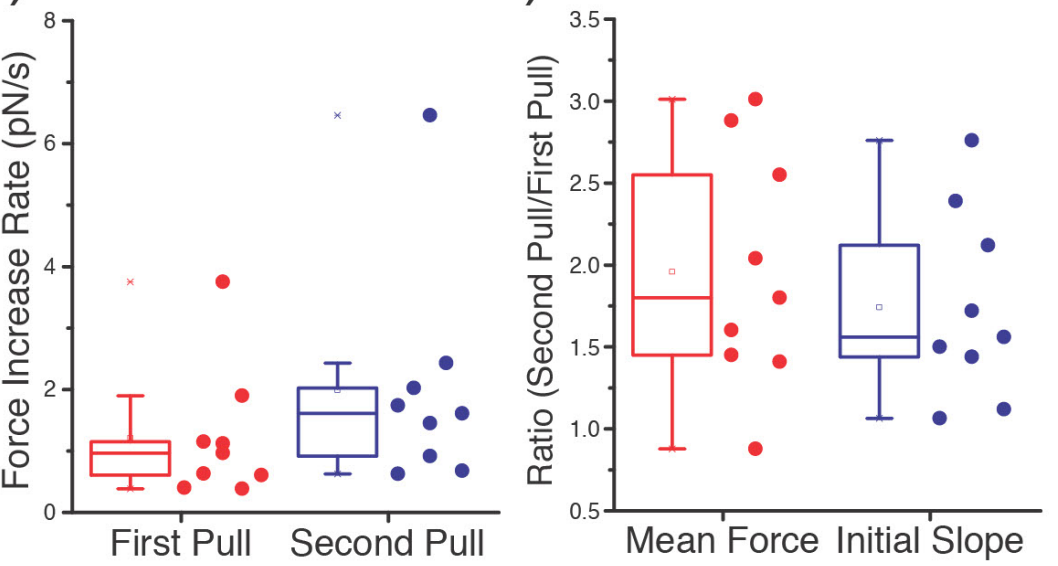
547 Figure 5. A computational model predicts a partially reflective end barrier against 548 PRC1 diffusion

549 (A) Schematic depicting parameters used in computational simulations. PRC1 molecules 550 bind to the microtubule lattice on sites with $8 \mathrm{~nm}$ periodicity, and can diffuse with rate $551 k_{\text {diffuse }}(F)$, detach from the microtubule with rate $k_{\text {detach }}(F)$, and diffuse off of microtubule 552 ends with rate $k_{\text {end. }}$. Force across each PRC1 molecule is calculated by $F=k_{\text {stiffness }}{ }^{*} \Delta x$.

553 (B) Effect of varying $k_{\text {end }}$ relative to $k_{\text {diffuse. }}$. For each parameter set, 20 simulations were 554 run, and values were averaged. Force versus overlap length are shown for a range of kend 555 values. (C) Force data from (B) plotted as a function of engaged PRC1 crosslinking 556 molecules. (D) Effect of varying $k_{\text {diffuse }}$ while setting $k_{\text {detach }}=0.002 \mathrm{~s}^{-1}$. Force versus 557 overlap length are shown for a range of $k_{\text {diffuse }}$ values $\left(20,000 \mathrm{~nm}^{2} / \mathrm{s}, 40,000 \mathrm{~nm}^{2} / \mathrm{s}\right.$, $558100,000 \mathrm{~nm}^{2} / \mathrm{s}$ ). (E) Effect of varying $k_{\text {detach }}$ while setting $k_{\text {diffuse }}=40,000 \mathrm{~nm}^{2} / \mathrm{s}$. Force 559 versus overlap length are shown for a range of $k_{\text {detach }}$ values $\left(0.002 \mathrm{~s}^{-1}, 0.005 \mathrm{~s}^{-1}, 0.01 \mathrm{~s}^{-}\right.$ $560{ }^{1}$, and $\left.0.05 \mathrm{~s}^{-1}\right)$. (F) Numerical simulations were performed using parameter values $k_{\text {detach }}$ $561=0.002 \mathrm{~s}^{-1}, k_{\text {diffuse }}=40,000 \mathrm{~nm}^{2} / \mathrm{s}$, and $k_{\text {end }}=75$. Averaged force values from $\mathrm{N}=20$ 562 independent simulations were calculated and plotted as a function of PRC1 crosslinking 563 molecules. (G) Slopes of data from (F) are plotted as a function of sliding velocity. (H) A 564 schematic model describing how resistive forces increase with both sliding velocity and 565 crosslinker number. 


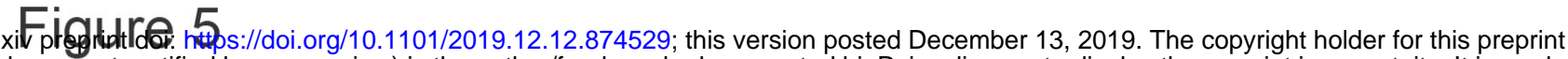
(which was not certified by peer review) is the author/funder, who has granted bioRxiv a license to display the preprint in perpetuity. It is made available under aCC-BY-NC-ND 4.0 International license.

A)

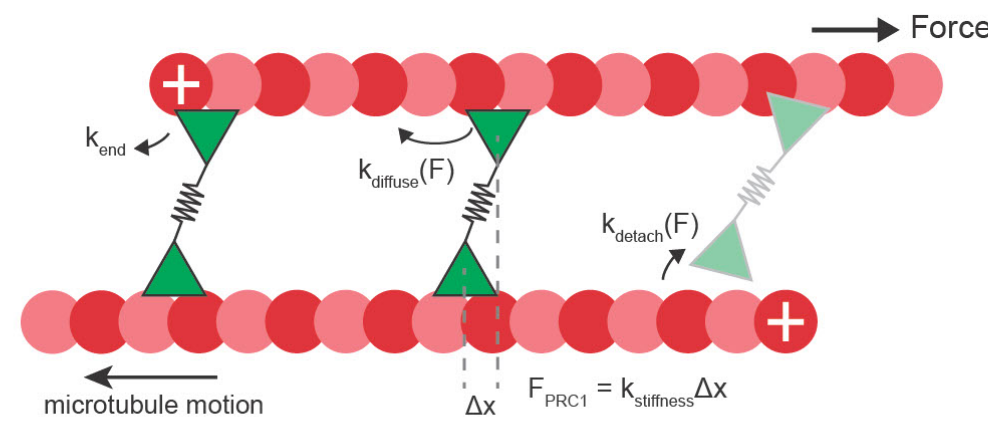

B)

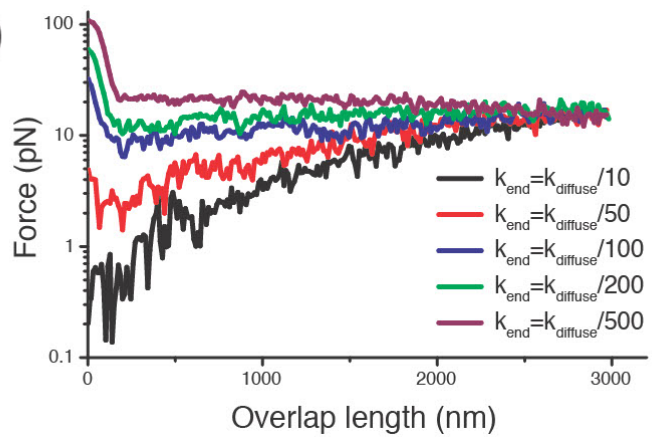

D)

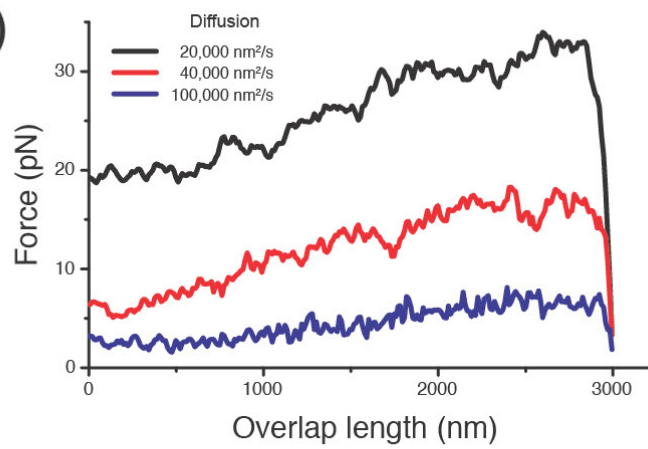

F)

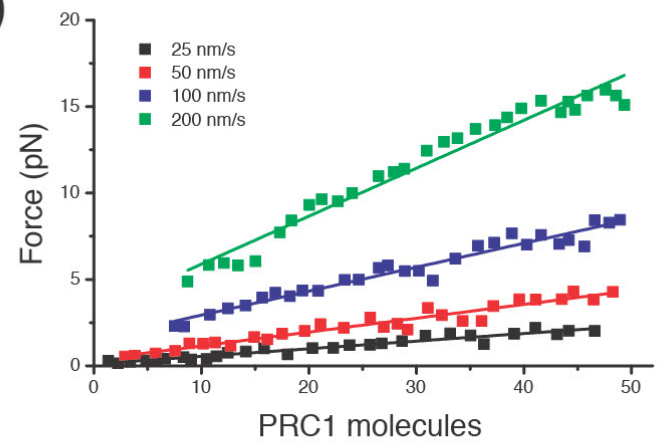

C)

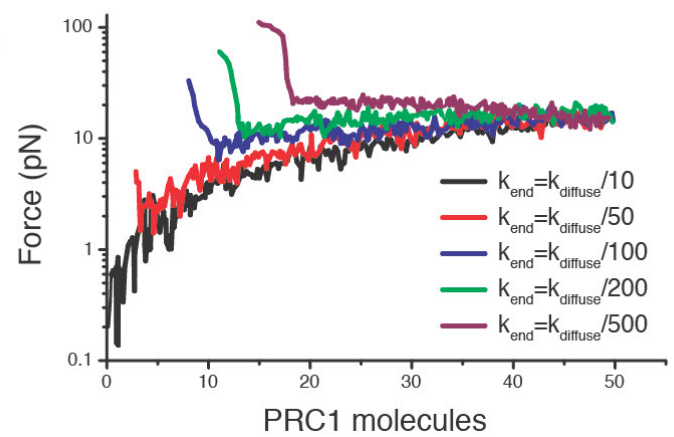

E)

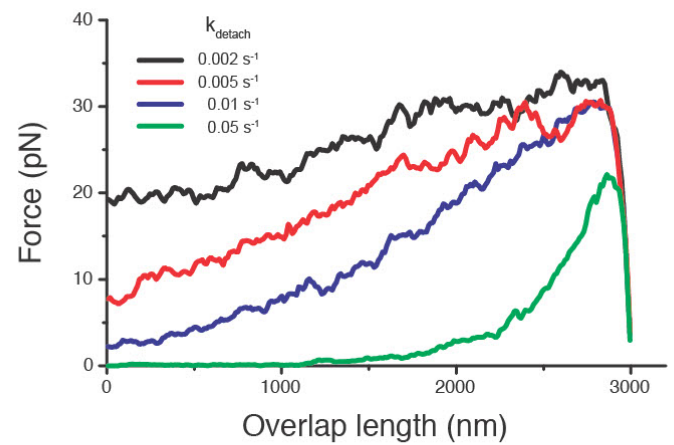

G)

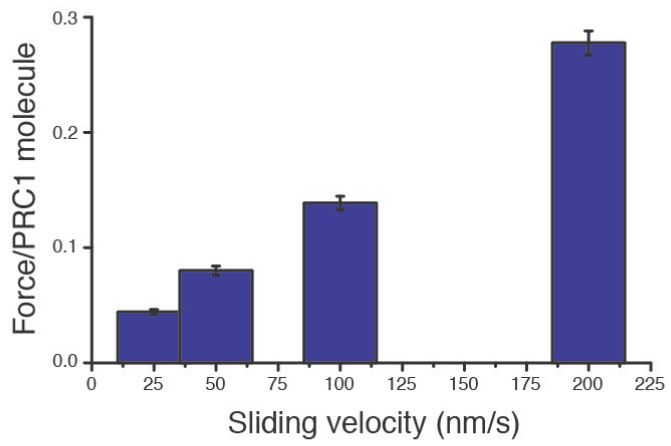

H)

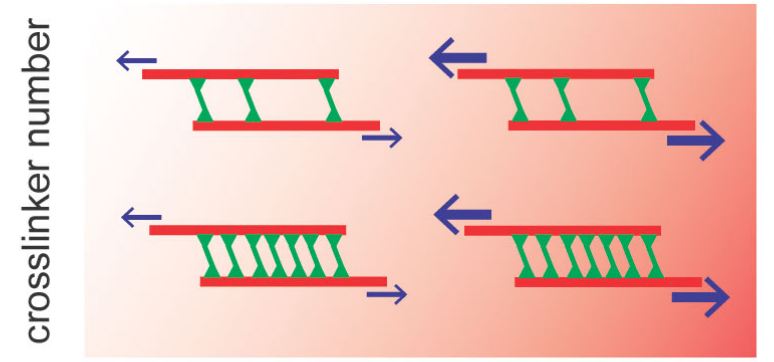

sliding velocity 


\section{Supplementary Figure 1. Direct Measurement of GFP-PRC1 Fluorescence Signal}

\section{Reduction due to Photobleaching.}

568 Assay set up similar to description in Figure 1 without kinesin coated bead or stage 569 motion. (A) Time series of fluorescent images acquired via TIRF microscopy. GFP-PRC1 570 and individual microtubule channels are shown along with composite image showing

571 every $3^{\text {rd }}$ frame ( $\sim 5.25$ second interval). Scale bar $=2$ microns. (B) Linescan analysis from

572 GFP channel. GFP intensities at select time points are plotted against length, with 573 corresponding time points noted on kymograph inset (blue $=0 \mathrm{~s}$, green $=26 \mathrm{~s}$, yellow $=$

$57450 \mathrm{~s}$, orange $=74 \mathrm{~s}$, red $=98 \mathrm{~s}$ ). Kymograph scale bars: vertical $=17 \mathrm{~s}$, horizontal $=0.5$ 575 microns. (C) GFP signals within overlap regions were integrated and normalized to their 576 initial values and are binned at 10 second intervals. Data from 9 individual measurements 577 were averaged. Error bars $=$ SE. 
bioRxiv preprint doi: https://doi.org/10.1101/2019.12.12.874529; this version posted December 13, 2019. The copyright holder for this preprint (which was not certified by peer review) is the author/funder, who has granted bioRxiv a license to display the preprint in perpetuity. It is made available under aCC-BY-NC-ND 4.0 International license.

\section{Figure S1}

A)

microtubule GFP-PRC1 microtubule composite

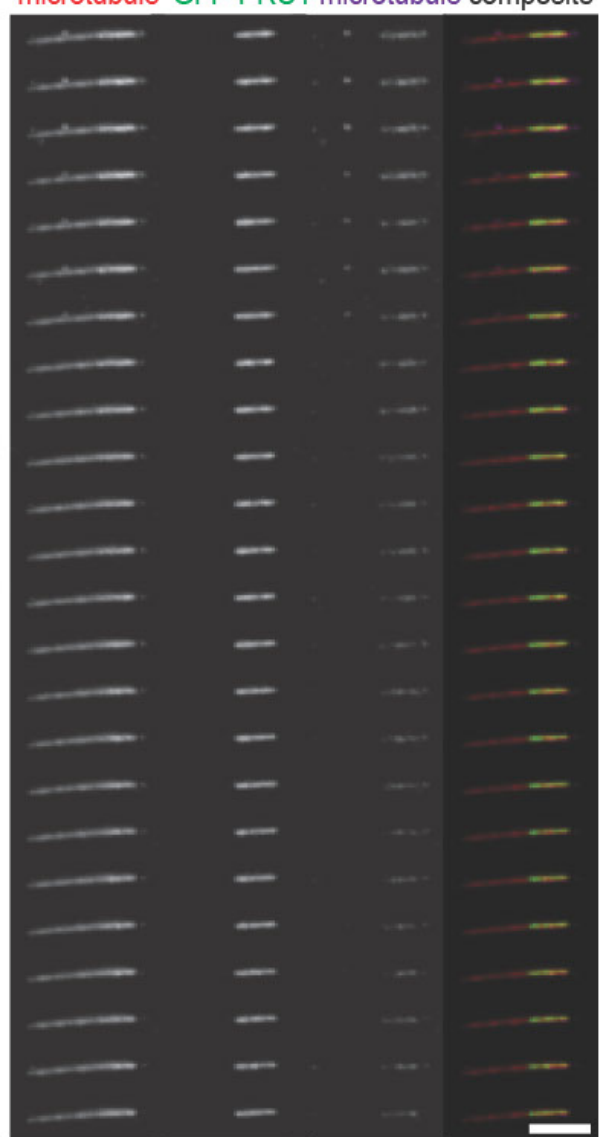

B)

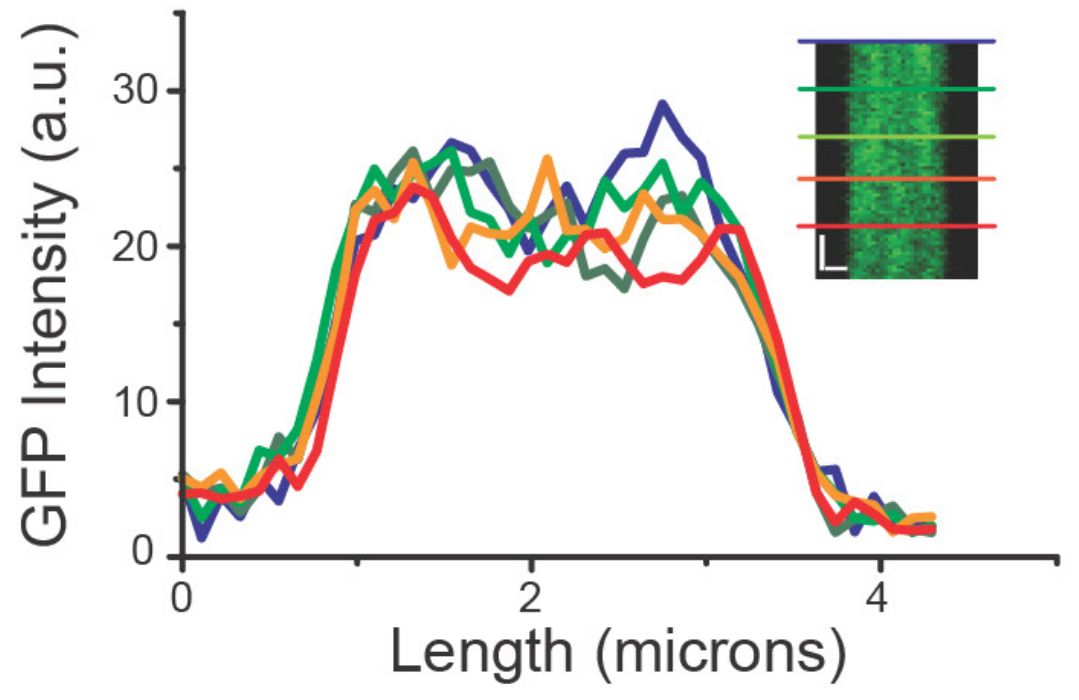

C)

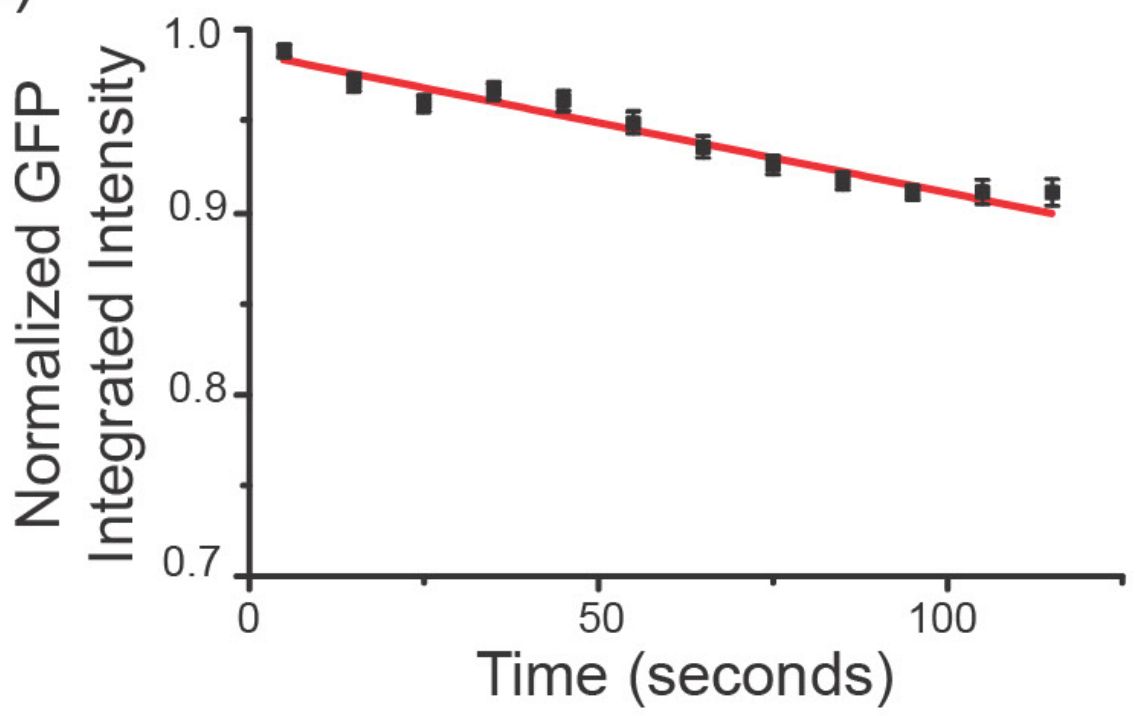




\section{Supplementary Figure 2. Example of Bead-microtubule detachment and 579 reattachment event}

580 (A) Representative force time series for bead sliding along microtubule assay. Red arrow 581 indicates kinesin coated bead detachment from rhodamine microtubule. Green Arrow 582 indicates kinesin coated bead re-attachment to rhodamine microtubule. (B) Kymographs 583 of corresponding force time series from top to bottom: free rhodamine microtubule, GFP584 PRC1, surface-immobilized Hilyte-647 microtubule, and composite of all three channels: 585 rhodamine (red), GFP-PRC1 (green), and Hilyte-647 (purple). Red arrow indicates 586 kinesin coated bead detachment from rhodamine microtubule. Green Arrow indicates 587 kinesin coated bead re-attachment to rhodamine microtubule. Kymograph scale bars: 588 vertical $=19 \mathrm{~s}$, horizontal $=2 \mu \mathrm{m}$. 
bioRxiv preprint doi: https://doi.org/10.1101/2019.12.12.874529; this version posted December 13, 2019. The copyright holder for this preprint (which was not certified by peer review) is the author/funder, who has granted bioRxiv a license to display the preprint in perpetuity. It is made available under aCC-BY-NC-ND 4.0 International license.

\section{Figure S2}
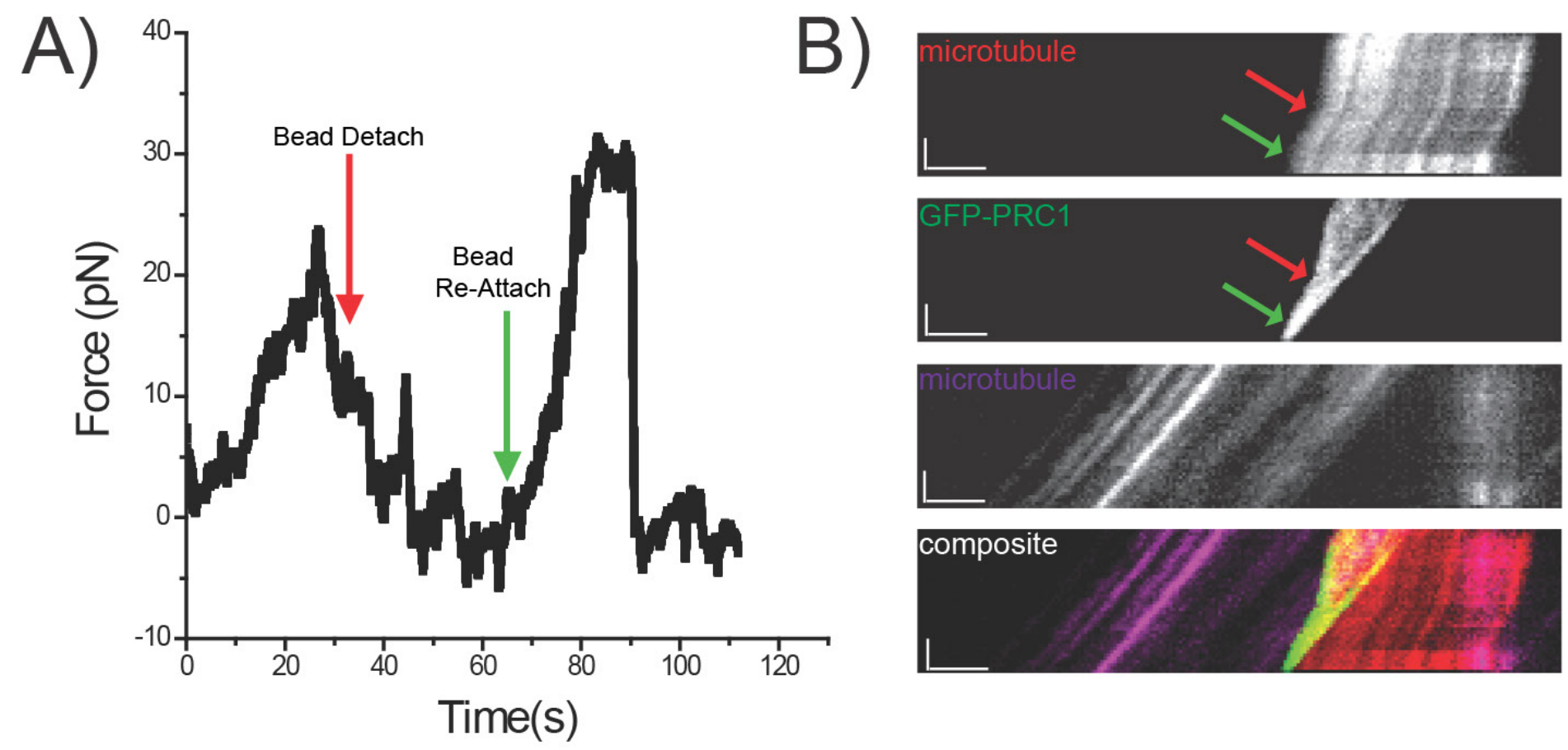

Time(s) 
589 Supplementary Figure 3. Confirmation of surface microtubule immobilization 590 during sliding event

591 (A) Representative force time series for bead sliding along microtubule assay. Green 592 arrow indicates start of bundle pull and red arrow indicates bundle separation. (B) 593 Kymographs of corresponding force time series from top to bottom: free rhodamine 594 microtubule, GFP-PRC1, surface-immobilized Hilyte-647 microtubule, and composite of 595 all three channels: rhodamine (red), GFP-PRC1 (green), and Hilyte-647 (purple). Green 596 arrow indicates start of bundle pull and red arrow indicates bundle separation. Yellow line 597 highlights trajectory of surface bound HiLyte647 microtubule and corresponds to a 598 measured velocity of $50 \mathrm{~nm} / \mathrm{s}$. Kymograph scale bars: vertical $=19 \mathrm{~s}$, horizontal $=2 \mu \mathrm{m}$. 
bioRxiv preprint doi: https://doi.org/10.1101/2019.12.12.874529; this version posted December 13, 2019. The copyright holder for this preprint (which was not certified by peer review) is the author/funder, who has granted bioRxiv a license to display the preprint in perpetuity. It is made available under aCC-BY-NC-ND 4.0 International license.

\section{Figure S3}

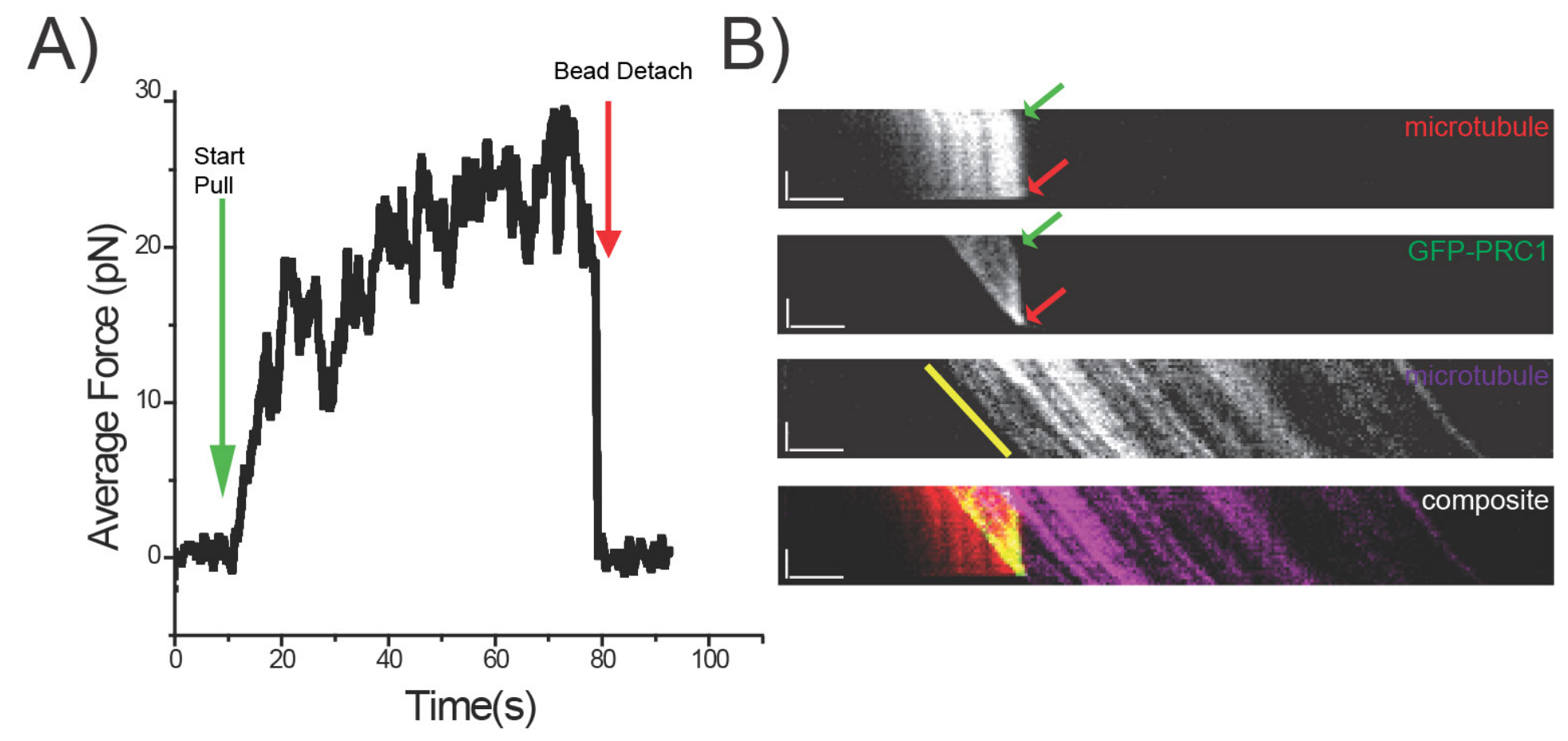


Methods

600

601

602

\section{Protein expression and purification}

603 We first generated an GFP-tagged PRC1 isoform 2 (aa:1-606) construct by deleting 14aa 604 from near the C-terminus of PRC1 isoform 1 (aa:1-620) using Quikchange mutagenesis 605 (Agilent). The PRC1 isoform 2 gene was then in a pET-DUET plasmid containing an N606 terminal histidine tag followed by a Tobacco Etch Virus (TEV) cleavage site and an EGFP 607 sequence inserted in between the TEV cleavage site and the $\mathrm{N}$-terminus of PRC1 isoform 6082 with a 'AAA' linker sequence just after the eGFP (Subramanian et al., 2010). GFP-PRC1 609 isoform 2 proteins were expressed via BL21(DE3) Rosetta Escherichia coli cells 610 (Novagen). Full length PRC1 was expressed for 4 hours at $18^{\circ} \mathrm{C}$ after induction with $6110.5 \mathrm{mM}$ IPTG. Cells were lysed via sonication in lysis buffer $(1 \mathrm{mg} / \mathrm{mL}$ lysozyme, $50 \mathrm{mM}$ 612 phosphate $(\mathrm{pH} 8.0), 10 \mathrm{mM}$ imidazole, and 1\% Igepal and HALT protease inhibitor 613 (Pierce), $2 \mathrm{mM}$ TCEP Bond Breaker, $2 \mathrm{mM}$ Benz-HCl, $1 \mathrm{mM}$ PMSF). The lysate was 614 clarified by ultracentrifugation and the supernatant was incubated with Ni-NTA for 1 hour 615 at $4^{\circ} \mathrm{C}$ (G Biosciences). The resin was then washed with Wash Buffer (50 mM phosphate, 616 (pH 8), $500 \mathrm{mM} \mathrm{KCl}, 10 \mathrm{mM}$ imidazole, $0.1 \%$ tween, $0.5 \mathrm{mM}$ TCEP, $1 \mathrm{mM} \mathrm{PMSF}$ ) and 617 the protein was then eluted using Elution Buffer (50 mM phosphate (pH 7.0), $250 \mathrm{mM}$ 618 imidazole, $150 \mathrm{mM} \mathrm{KCl}, 0.5 \mathrm{mM}$ TCEP). Elute was pooled and concentrated to volume of 619 approximately $1 \mathrm{~mL}$. Next, 1/30 w/w Pro TEV protease (PROMEGA), $1 \mathrm{mM}$ DTT, and 50 $620 \mu \mathrm{L}$ of Pro TEV 20X buffer were added and the sample incubated in a $30^{\circ} \mathrm{C}$ water bath for 62115 mins before overnight dialysis at $4^{\circ} \mathrm{C}$ in Gel Filtration Buffer (1X BRB80 (pH 6.8), 150 $622 \mathrm{mM} \mathrm{KCl}, 10 \mathrm{mM} \beta \mathrm{ME}$ ). Size exclusion chromatography (Superose-6 increase 10/300 623 column, GE Healthcare) was then performed in Gel Filtration Buffer with a Shimadzu High 624 Performance Liquid Chromatograph. After collecting peak fractions and concentrating to $625 \sim 0.5 \mathrm{mg} / \mathrm{mL}$, sucrose was added to $35 \% \mathrm{w} / \mathrm{v}$ before flash freezing in liquid nitrogen. 


\section{Kinesin-1 K439}

631 Plasmid encoding truncated kinesin-1 construct (K439) with a C-terminally fused EB1 sequence to create stable dimers and a His8 tag (Woll et al., 2018) was generously donated by the Dr. Susan Gilbert lab. The protein expression protocol was identical to 634 PRC1, except induction occurred overnight for 18 hours at $16^{\circ} \mathrm{C}$. The pellet was resuspended in lysis buffer (10 $\mathrm{mM} \mathrm{NaPO}_{4}(\mathrm{pH} 7.2), 300 \mathrm{mM} \mathrm{NaCl}, 2 \mathrm{mM} \mathrm{MgCl}, 0.1 \mathrm{mM}$ EGTA, 10 mM PMSF, 1 mM DTT, 0.2 mM ATP, 1 mg/mL lysozyme, 30 mM imidazole, 3 $\mu \mathrm{L}$ benzonase (Novagen) and sonicated. The lysate was clarified by ultracentrifugation and the supernatant incubated with Ni-NTA for 1 hour at $4^{\circ} \mathrm{C}$ (G Biosciences). The resin was then washed with Wash Buffer (20 mM NaPO 4 (pH 7.2), 300 mM NaCl, 2 mM MgCl , $0.1 \mathrm{mM}$ EGTA, $0.02 \mathrm{mM}$ ATP, $50 \mathrm{mM}$ Imidazole) and the protein was then eluted using Elution Buffer (20 mM NaPO 4 ( pH 7.2), 300 mM NaCl, 2 mM MgCL2, 0.1 mM EGTA, 1 mM DTT, $0.02 \mathrm{mM}$ ATP, $400 \mathrm{mM}$ imidazole). Elute was pooled, concentrated to volume of approximately $1 \mathrm{~mL}$. The protein was then dialyzed overnight at $4^{\circ} \mathrm{C}$ in Dialysis Buffer (20 mM HEPES (pH 7.2), 300 mM NaCl, 0.1 mM EGTA, 0.1 mM EDTA, 5 mM MgAc, 50 $\mathrm{mM} \mathrm{KAc}, 1 \mathrm{mM}$ DTT. The next day the protein was dialyzed for 1 hour at $4^{\circ} \mathrm{C}$ in Buffer 1 (20 mM HEPES (pH 7.2), 200 mM NaCl, 0.1 mM EGTA, 0.1 mM EDTA, 5 mM MgAc, 50 $\mathrm{mM} \mathrm{KAc}, 1 \mathrm{mM}$ DTT) followed by dialysis for 1 hour at $4^{\circ} \mathrm{C}$ in Buffer 2 (20 mM HEPES (pH 7.2), 150 mM NaCl, 0.1 mM EGTA, 0.1 mM EDTA, 5 mM MgAc, 50 mM KAc,1 mM DTT)

649 Further purification was performed using size exclusion chromatography (Superose-6 increase, GE Healthcare) using Shimadzu High Performance Liquid Chromatograph. Peak fractions were pooled and dialyze for 3 hours at $4^{\circ} \mathrm{C}$ of Buffer $3(20 \mathrm{mM}$ HEPES (pH 7.2), 100 mM NaCl, 0.1 mM EGTA, 0.1 mM EDTA, 5 mM MgAc, 50 mM KAc,1 mM DTT, $5 \%$ Sucrose). Protein was flash frozen in liquid nitrogen.

\section{Microtubule preparation}

656 Microtubule tubulin reagents were purchased from Cytoskeleton, Inc. Microtubules for 657 surface-immobilization were generated via mixture of HiLyte 647 tubulin (TL670M), 658 biotinylated tubulin (T333P), and unmodified tubulin (T240) at a ratio of 1:1:20 along with $6591 \mathrm{mM}$ GMPCPP. Microtubules were polymerized at $37^{\circ} \mathrm{C}$ for 1 hour before clarification and 660 stabilization in 30uM Taxol following published protocols (Shimamoto 2015). 'Free' 
661 microtubules for attachment to optically trapped beads were generated via mixture of 662 rhodamine tubulin (TL590M) and unmodified tubulin at a ratio of 1:20 along with $1 \mathrm{mM}$ 663 GMPCPP, and were polymerized, clarified, and stabilized in 30uM Taxol following similar 664 protocols.

665

\section{Coating Beads with K439}

667 One-micron diameter streptavidin-coated polystyrene beads (Polysciences Inc., \#24162-

668 1) were coated with biotin-conjugated His 6 antibody (Invitrogen) followed by washing and storage in 2mg/mL alpha-Casein solution made in BRB80 (80 mM K-PIPES, $1 \mathrm{mM} \mathrm{MgCl}_{2}$, 1mM EGTA, 2mM DTT (pH 6.8)). K439 samples were diluted into BRB80 mixed at 1:1 ratio with coated polystyrene beads. Samples were placed on a rotor for 30 minutes at $4^{\circ} \mathrm{C}$ followed by sonication for 10 minutes.

\section{Flow Chamber Construction:}

675 The flow chamber design and assay preparation were modified from a previously 676 described protocol (Shimamoto et al., 2015). Anti-parallel microtubule bundles were 677 constructed using passivated glass coverslips coated with SVA-PEG at a ratio of 50 678 PEG:1 biotin-PEG. All reagents were prepared with BRB80 buffer. Following each reagent flow-in and incubation, a flush with $\sim 3$ chamber volumes of BRB80 was performed. Reagents were introduced stepwise with the following order and incubation times: (1) $0.5 \mathrm{mg} / \mathrm{mL}$ neutravidin, 2 minutes; (2) $0.5 \mathrm{mg} / \mathrm{mL}$ alpha casein surface block, 3 minutes; (3) HiLyte-647 biotinylated microtubules with $0.2 \mathrm{mg} / \mathrm{mL}$ alpha casein with no additional incubation and immediate flush; (4) $1 \mathrm{nM}$ GFP-PRC1 with $0.2 \mathrm{mg} / \mathrm{mL}$ alpha casein, 2 minutes; (5) Rhodamine 561 microtubules with $0.2 \mathrm{mg} / \mathrm{mL}$ alpha casein, 5 minutes, and the corresponding chamber flush included $1 \mathrm{mM}$ TCEP bond breaker solution in BRB80; (6) $1 \mu \mathrm{L}$ of K439 coated beads, $1 \mathrm{mM}$ of TCEP bond breaker, 0.2

$687 \mathrm{mg} / \mathrm{mL}$ alpha casein, $70 \mathrm{mM} \mathrm{KCl}$, Oxygen Scavenging System $(4.5 \mathrm{mg} / \mathrm{ml}$ glucose, 350 $688 \mathrm{U} / \mathrm{ml}$ glucose oxidase, $34 \mathrm{U} / \mathrm{ml}$ catalase, $1 \mathrm{mM}$ DTT). The chamber was then sealed with 689 clear nail polish prior to experiments. 


\section{Image Acquisition:}

693 Microtubule bundles were imaged using three-channel TIRF microscopy using the 694 following laser lines and exposure times: HiLyte-647 microtubules: $640 \mathrm{~nm}$ laser (60\% 695 power, $200 \mathrm{~ms}$ exposure); GFP-tagged PRC1: $488 \mathrm{~nm}$ laser (30\% power, $100 \mathrm{~ms}$ 696 exposure); and rhodamine microtubules: $561 \mathrm{~nm}$ laser (30\% power, $100 \mathrm{~ms}$ exposure). 697 Images were acquired using a Photometric Prime 95B camera controlled with Nikon NIS 698 Elements software at overall acquisition rates of one frame per $\sim 1.75$ seconds. Prior to 699 analysis, images were visually screened to ensure that there were no additional 700 interactions with microtubules from other bundles. Analysis of fluorescent data and 701 generation of intensity linescan data sets were performed using a combination of FIJI 702 (ImageJ) tools and custom-written LabVIEW software.

\section{Force Data Acquisition}

705 The optical tweezers system was constructed based on a fiber-coupled infrared laser 706 (1064 nm, 10W, IPG Photonics) and a position-sensitive detector (Thorlabs PDQ80A). 707 The laser beam power was controlled using an AOM (AA Optoelectronics MTS80-A3708 1064Ac) and expanded using a custom-built telescope (Thorlabs optics). The beam was 709 then passed through a 1:1 telescope and merged into the microscope's light path using 710 a dichroic IR filter (z900dscp, Chroma) mounted on a secondary turret located above the

711 fluorescence turret. The beam was introduced into the back aperture of the objective and 712 focused to a diffraction-limited spot such that a micron-sized bead could be trapped 713 approximately 500nm above the coverslip surface. A high NA 100× objective (1.49 NA; 714 CFI Plan Apo TIRF) was used for establishing both stable optical trapping and high715 resolution fluorescence imaging. Coverslip position was controlled with sub-nanometer 716 precision using a closed-loop three-axis piezo stage (Nano LPS-200, Mad City Labs). To 717 monitor the displacement of the bead held in the optical tweezers, the laser beam passing 718 through the sample plane was collected using an oil-immersion condenser (MEL41410, 719 Nikon) and reflected away from the microscope's imaging path by a dichroic IR mirror 720 (DMSP805L, Thorlabs). After passing through a long-pass optical filter, the beam was 721 projected onto the quadrant photodiode detector placed conjugate to the back-focal plane 722 of the objective. The signals from each photodiode quadrant were amplified and recorded 
723 using an in-house developed LabVIEW program via an AD converter (PCle-6363,

724 National Instruments). The displacement signal along each coordinate was obtained by

725 calculating the difference of the normalized signals between the adjacent quadrant pairs.

726 Bead position and force data were converted from raw voltage to physical units after

727 standard calibration methods were employed using custom-written LabView software.

728 Absolute force values were determined by introducing a constant offset such that zero-

729 force corresponded to force values recorded immediately upon bundle separation.

731 Computational Simulations

732 To simulate the resistive forces generated by ensembles of diffusive PRC1 crosslinkers

733 between two sliding microtubules, we modified a Monte-Carlo based technique previously

734 employed to model kinesin-5 stepping behavior (Shimamoto et al., 2015). Briefly,

735 individual PRC1 molecules can be defined as two microtubule binding domains

736 connected by a spring-like linker domain that allows for crosslinking of two individual

737 microtubules. For each MT-binding domain a diffusion parameter $k_{\text {diffuse }}$ can be assigned,

738 as well as a rate of detachment $k_{\text {detach, }}$ and both parameters can depend on applied force

739 via an Arrhenius-like term.

741 To describe the behavior of a PRC1 molecule which is crosslinking two microtubules, we

742 consider two independent MT-binding domains connected by a spring-like linker region

743 which stretches as the two heads become spatially separated as they move along the

744 microtubule surface. This parameter $k_{\text {stiffness }}$ is a simple linear spring constant term that

745 coarsely defines an energy penalty for the spatial separation of the MT-binding domains.

747 Distribution of forces across PRC1 molecules with optically trapped microtubule end

748 The central feature of our simulation is the balance of forces across the PRC1 molecules

749 with the optically trapped microtubule end serving as a linear spring providing resistance

750 to microtubule motion. For a given number of molecules, we require that the forces must

751 balance according to:

$$
F_{\text {trap }}=\sum F_{P R C 1}
$$


754 By defining the coordinates of discrete (i.e., $8 \mathrm{~nm}$ spacing) positions along the free ('top')

755 microtubule and the discrete positions along the immobilized 'bottom' microtubule, as well

756 as considering the position of the moving free microtubule end (which is equivalent to the

757 optical trap position, $\left.x_{\text {trap }}\right)$, we can rewrite this relationship as:

758

759

$$
-k_{\text {trap }} x_{\text {trap }}+\sum_{i}^{N}\left(k_{P R C 1} *\left[\left(x_{\text {i TOP }}-x_{\text {trap }}\right)-x_{\text {i воттом }}\right]\right)=0
$$

760

761

Solving for $x_{\text {trap }}$ yields:

762

763

764

$$
\begin{aligned}
& -\frac{k_{\text {trap }}}{k_{P R C 1}} x_{\text {trap }}-N x_{\text {trap }}+\sum_{i}^{N} x_{i \text { TOP }}-\sum_{i}^{N} x_{\text {i вотTом }}=0 \\
& \left(N+\frac{k_{\text {trap }}}{k_{P R C 1}}\right) x_{\text {trap }}=\sum_{i}^{N} x_{i \text { TOP }}-\sum_{i}^{N} x_{i \text { вотTом }} \\
& x_{\text {trap }}=\frac{\sum_{i}^{N} x_{i \text { TOP }}-\sum_{i}^{N} x_{i \text { воттом }}}{\left(N+\frac{k_{\text {trap }}}{k_{\text {PRC1 }}}\right)}
\end{aligned}
$$

The position of the microtubule end (trap position, $x_{\text {trap}}$ ) will therefore depend on the relative positions along the microtubules of each PRC1 MT-binding domain, the total number of molecules engaged in crosslinking, and both the trap and PRC1 linker domain stiffness.

Monte Carlo Simulations

773 Start at Time $=0 \mathrm{~s}$ with all PRC1 molecules in a fully relaxed state (i.e., with all Force PRC1

$774=0 \mathrm{pN}$ ) and uniformly spaced within the overlap region, as well as the condition that 775 Force $_{\text {trap }}=0 \mathrm{pN}$.

776 At each subsequent time step, perform the following:

777 1.) For each PRC1 molecule, check the attachment state of both the top and bottom heads. If the head is attached, detach if $\operatorname{rand}()<k_{\text {detach }}(F)^{*} \Delta t$. If $\operatorname{rand}()>$ Probability, do nothing and calculate attachment state of next molecule. 
2.) For each diffusive head:

a. Allow the head to diffuse in the direction of force application if $\operatorname{rand}()<k_{\text {diffuse }}{ }^{*} \exp \left(\mathrm{F}^{*}(4 \mathrm{~nm}) / \mathrm{kT}\right)$ or in the direction opposite force application if $\operatorname{rand}()>1-\left(k_{\text {diffuse }}{ }^{*} \exp \left(-\mathrm{F}^{*}(4 \mathrm{~nm}) / \mathrm{kT}\right)\right)$.

b. If adjacent site is occupied by another PRC1 molecule, do not allow head to diffuse onto the site; head remains at current site.

c. If PRC1 molecule is occupying the last available site on the microtubule lattice, allow it to diffuse off of microtubule end if $\operatorname{rand}()<k_{e n d}{ }^{*} \exp \left(\mathrm{F}^{*}(4\right.$ $\mathrm{nm}) / \mathrm{kT})$.

d. If none of the above conditions are met, allow the head's position to remain unchanged.

3.) Determine the number of PRC1 molecules that have both heads attached, $N_{\text {engaged. }}$

4.) Calculate the new position of the trap according to:

$$
x_{\text {trap }}=\frac{\sum_{1}^{N}\left(x_{\text {T TOP attach }} * x_{i \text { TOP }}\right)-\sum_{1}^{N}\left(x_{\text {ВотTOM attach }} * x_{\text {ВотTOM }}\right)}{N_{\text {engaged }}+\frac{k_{\text {trap }}}{k_{P R C 1}}}
$$

5.) Calculate the Force across each attached motor according to:

$$
\text { Force }_{i}=k_{P R C 1} * x_{\text {attach }_{i}} *\left(x_{\text {pos }_{\text {TOP } i}}-x_{\text {trap }}+x_{\text {pos }_{\text {BOTTOM_i }}}\right)
$$

6.) Increment the absolute position of the bottom ("surface-immobilized") microtubule by a distance Rate* $\Delta t$, where rate is selected from $\{25,50,100,200\} \mathrm{nm} / \mathrm{s}$. The overlap length is therefore reduced in an approximately linear manner until it reaches zero, at which point no PRC1 molecules are available to crosslink the microtubule pair and the force on the free microtubule as measured by the bead falls to zero.

Repeat steps 1-6 with updated values for Force per PRC1 molecule for desired length of time (typically 30-90 seconds). 